\title{
ADAPTIVE BOUNDARY-ELEMENT METHODS FOR TRANSMISSION PROBLEMS
}

\author{
CARSTEN CARSTENSEN ${ }^{1}$ and ERNST P. STEPHAN ${ }^{2}$
}

(Received 20 September 1993; revised 2 January 1995)

\begin{abstract}
In this paper we present an adaptive boundary-element method for a transmission problem for the Laplacian in a two-dimensional Lipschitz domain. We are concerned with an equivalent system of boundary-integral equations of the first kind (on the transmission boundary) involving weakly-singular, singular and hypersingular integral operators. For the h-version boundary-element (Galerkin) discretization we derive an a posteriori error estimate which guarantees a given bound for the error in the energy norm (up to a multiplicative constant). Then, following Eriksson and Johnson this yields an adaptive algorithm steering the mesh refinement. Numerical examples confirm that our adaptive algorithms yield automatically good triangulations and are efficient.
\end{abstract}

\section{Introduction}

The problem of constructing an adaptive mesh-refining procedure is of practical importance in the numerical analysis of partial differential equations. Since the pioneering work of Babuška and Miller [1] and Eriksson and Johnson [10, 11] adaptive finite-element methods are well established. We extend our analysis of adaptive boundary-element methods $[3,4]$ to boundary-integral equations for elliptic interface problems. We prove a posteriori error estimates for the Galerkin boundary-element solution and develop various adaptive mesh-refinement algorithms.

In this paper we convert the interface problem for harmonic functions $u_{1}$ in $\Omega_{1}$ and $u_{2}$ in $\Omega_{2}$ into a system of boundary-integral equations on the interface boundary $\Gamma$ between the bounded interior domain $\Omega_{1}$ and the unbounded exterior $\Omega_{2}:=\mathbb{R}^{2} / \bar{\Omega}_{1}$. We prescribe the jumps $u_{1}-u_{2}$ and $\frac{\partial u_{1}}{\partial n}-\frac{\partial u_{2}}{\partial n}$ on the interface $\Gamma\left(\frac{\partial}{\partial n}\right.$ denotes the normal

\footnotetext{
The work is partly supported by DFG research group at the University of Hannover ${ }^{1}$ Mathematisches Seminar, Universität Kiel, Ludewig-Meyn-Str. 4, D-24098 Kiel, FRG. Email: cc@numerik.uni-kiel.de ${ }^{2}$ Institut für Angewandte Mathematik, UNI Hannover, D-30167 Hannover, FRG.

(C) Australian Mathematical Society, 1997, Serial-fee code 0334-2700/96
} 
derivative) and obtain a system of boundary integral equations of the first kind for the unknown traces $\left.u_{1}\right|_{\Gamma}$ and $\left.\frac{\partial u_{1}}{\partial n}\right|_{\Gamma}$. Our solution procedure for the original interface problem consists now in solving this system of integral equations for $\left.u_{1}\right|_{\Gamma}$ and $\left.\frac{\partial u_{1}}{\partial n}\right|_{\Gamma}$, then using the values for the jumps to compute $\left.u_{2}\right|_{\Gamma}$ and $\left.\frac{\partial u_{2}}{\partial n}\right|_{\Gamma}$ and finally computing $u_{i}(x)$ for $x \in \Omega_{i}$ with the representation formula as a combination of single-layer and double-layer potentials acting on the traces $\left.u_{i}\right|_{\Gamma}$ and $\left.\frac{\partial u_{i}}{\partial n}\right|_{\Gamma}$. This procedure is explained in detail in [7], where we analyzed the solvability of the system of boundary integral equations (see also Theorem 1 below). This system, (4) below, consists of the operators of the single-layer potential and the double-layer potential and their normal derivatives. Thus, the corresponding integral operators have weakly-singular, singular and hypersingular kernels, respectively. The system can be solved approximately by the Galerkin method (see [7]). For the implementation of this method see Section 7 below. Since our system of boundary-integral operators is a strongly-elliptic system of pseudodifferential operators on $\Gamma$ [7] (note that the single-layer potential operator $V$ is a strongly-elliptic operator from $H^{-1 / 2}(\Gamma)$ into $H^{1 / 2}(\Gamma)$ and the normal derivative of the double layer potential $W$ is a strongly elliptic from $H^{1 / 2}(\Gamma)$ into $H^{-1 / 2}(\Gamma)$ ) the Galerkin boundary-element solution converges quasioptimally (see Theorem 2 below) towards the exact solution of our system of integral equations on $\Gamma$.

It was shown in [7] that the rate of convergence of the corresponding boundaryelement Galerkin solution is restricted by the regularity of the exact solution of the boundary-integral equations. In [7], the h-version of the boundary-element method was analyzed on quasi-uniform meshes where the trace of the solution of the original interface problem was approximated by piecewise-linear continuous elements and its normal derivative by piecewise-constant functions. Here, we present an adaptive scheme for the h-version where a local mesh refinement is performed and therefore higher accuracy is achieved for the computed Galerkin solution than with quasiuniform meshes. Our adaptive refinement strategy is related to Eriksson and Johnson's approach for FEM and is based on an a posteriori estimate (Theorem 5) which endows the weighted residues in the $H^{1}(\Gamma) \times L^{2}(\Gamma)$-norm. The proof of the a posteriori error estimate is different from that in $[3,4]$. Applying this error indicator our adaptive process is steered by first computing a new mesh and then a corresponding Galerkin solution which is afterwards used to create a new mesh via local residues and so forth. This technique had turned out to be successful for integral equations of the first kind (with weakly singular and hypersingular kernels) in [4]. Our numerical results show improved convergence of the Galerkin solution and underline the efficiency of the adaptive method. 


\section{The transmission problem}

In this section we study the solvability of the boundary integral equation for a transmission problem of harmonic functions in $\mathbb{R}^{2}$. First let us introduce some notation before we formulate the transmission problem. Let $\Omega_{1} \subseteq \mathbb{R}^{2}$ be a bounded domain with Lipschitz boundary $\Gamma$ and let $\Omega_{2}:=\mathbb{R}^{2} \backslash \bar{\Omega}_{1}$ be its complement. Let $H^{s}(\Gamma)$ be the usual Sobolev spaces on the interface $\Gamma[15]$ such that $H^{0}(\Omega)=L^{2}(\Gamma)$ and $H^{-s}(\Gamma)$ is the dual space of $H^{s}(\Gamma), s>0$, with respect to the duality $($,$\rangle which is$ defined for smooth functions $u, v$ by

$$
\langle u, v\rangle=\int_{\Gamma} u \cdot v d s .
$$

Note that $H^{1 / 2}(\Gamma)$ is the trace space of $H^{1}\left(\Omega_{1}\right)$ and $H_{l o c}^{1}\left(\Omega_{2}\right)$.

Then the transmission problem under consideration reads as follows:

Given $f \in H^{1 / 2}(\Gamma)$ and $g \in H^{-1 / 2}(\Gamma)$ find

$$
\left(u_{1}, u_{2}\right) \in H^{1}\left(\Omega_{1}\right) \times H_{l o c}^{1}\left(\Omega_{2}\right)
$$

with

$$
\Delta u_{j}=0 \quad \text { in } \Omega_{j} \quad(j=1,2)
$$

and

$$
u_{1}=u_{2}+f, \quad \frac{\partial u_{1}}{\partial n}=\frac{\partial u_{2}}{\partial n}+g \quad \text { on } \Gamma
$$

such that there exists $b \in \mathbb{R}$ with

$$
\lim _{|x| \rightarrow \infty}\left(u_{2}(x)-\frac{b}{2 \pi} \log |x|\right)=0 .
$$

REMARK 1. (i) The trace $\left.u_{j}\right|_{\Gamma}$ is defined for $\left.u_{j}\right|_{\Gamma} \in H_{l o c}^{1}\left(\Omega_{j}\right)$ with the well-known trace theorems.

(ii) The normal derivatives $\frac{\partial u_{j}}{\partial n}, n$ being the normal on $\Gamma$ pointing from $\Omega_{1}$ into $\Omega_{2}$, is defined via Green's formula

$$
\int_{\Omega_{j}}(v \cdot \Delta u+\nabla v \cdot \nabla u) d x=(-1)^{j+1}\left\langle\left.\frac{\partial u_{j}}{\partial n}\right|_{\Gamma},\left.v\right|_{\Gamma}\right\rangle \quad(j=1,2),
$$

where $u_{j} \in H_{l o c}^{1}\left(\Omega_{j}\right)$ and $v \in H^{1}\left(\Omega_{j}\right)$ have compact support defining $\left.\frac{\partial u_{j}}{\partial n}\right|_{\Gamma} \in$ $H^{-1 / 2}(\Gamma)$ [7]. 
(iii) The condition (3) is given detailed consideration in [7].

(iv) Transmission problems of this type are analyzed in [7] in a quite general setting including scattering problems arising in electrostatics. The transmission problem (1)-(3) is a model problem in this field and the results below carry over to other transmission problems in $[7,8]$.

Following [7] we rewrite the transmission problem as a boundary-integral equation where we use some pseudodifferential operators. Given $v \in H^{1 / 2}(\Gamma)$ and $\phi \in$ $H^{-1 / 2}(\Gamma)$, define for $z \in \Gamma$ the boundary-integral operators

$$
\begin{aligned}
(V \phi)(z) & :=-\frac{1}{\pi} \int_{\Gamma} \phi(\zeta) \log |z-\zeta| d s_{\zeta}, \\
(K v)(z) & :=-\frac{1}{\pi} \int_{\Gamma} v(\zeta) \frac{\partial}{\partial n_{\zeta}} \log |z-\zeta| d s_{\zeta}, \\
\left(K^{\prime} \phi\right)(z) & :=-\frac{1}{\pi} \int_{\Gamma} \phi(\zeta) \frac{\partial}{\partial n_{z}} \log |z-\zeta| d s_{\zeta}, \\
(W v)(z) & :=\frac{1}{\pi} \frac{\partial}{\partial n_{z}} \int_{\Gamma} v(\zeta) \frac{\partial}{\partial n_{\zeta}} \log |z-\zeta| d s_{\zeta},
\end{aligned}
$$

which are bounded and linear in the spaces (see $[6,7])$

$$
\begin{gathered}
V: H^{s-1 / 2}(\Gamma) \rightarrow H^{s+1 / 2}(\Gamma), \\
K: H^{s+1 / 2}(\Gamma) \rightarrow H^{s+1 / 2}(\Gamma), \\
K^{\prime}: H^{s-1 / 2}(\Gamma) \rightarrow H^{s-1 / 2}(\Gamma), \\
W: H^{s+1 / 2}(\Gamma) \rightarrow H^{s-1 / 2}(\Gamma),
\end{gathered}
$$

where (since we allowed $\Gamma$ to be a Lipschitz boundary) $s \in[-1 / 2,1 / 2]$. Moreover the single-layer potential $V$ is symmetric, the double-layer potential $K$ has the dual $K^{\prime}$ and the hypersingular operator $W$ is symmetric. Note that $V$ and $W$ are strongly elliptic in the sense that they satisfy a Gårding inequality (in the above spaces with $s=0$; see [7]).

Define $H: H^{1 / 2}(\Gamma) \times H^{-1 / 2}(\Gamma) \rightarrow H^{1 / 2}(\Gamma) \times H^{-1 / 2}(\Gamma)$ by

$$
H:\left(\begin{array}{cc}
-K & V \\
W & K^{\prime}
\end{array}\right)
$$

The operator $H$ is linear, bounded and a Fredholm operator of index zero [7]. The following theorem describes the connection between the operator $H$ and the original problem (1)-(3).

THEOREM 1. (a) The transmission problem (1)-(3) is equivalent to the boundary 
integral equation

$$
H\left(\begin{array}{l}
v \\
\phi
\end{array}\right)=\frac{1}{2}(1+H)\left(\begin{array}{l}
f \\
g
\end{array}\right)
$$

in the following sense:

(i) If $\left(u_{1}, u_{2}\right) \in H^{1}\left(\Omega_{1}\right) \times H_{l o c}^{1}\left(\Omega_{2}\right)$ solves the transmission problem (1)-(3) then

$$
(v, \phi)=\left.\left(u_{1}, \frac{\partial u_{1}}{\partial n}\right)\right|_{\Gamma}
$$

solves the boundary integral equation (4).

(ii) If $(v, \phi) \in H^{1 / 2}(\Gamma) \times H^{-1 / 2}(\Gamma)$ solves the boundary integral equation (4) then the transmission problem (1)-(3) has a solution $\left(u_{1}, u_{2}\right)$ satisfying (5).

(b) The operator $H$ is bijective so that the transmission problem (1)-(3) as well as the integral equation (4) have unique solutions.

(c) Given $f \in H^{1}(\Gamma)$ and $g \in L^{2}(\Gamma)$ the solution $(v, \phi)$ of (4) satisfies $(v, \phi) \in$ $H^{1}(\Gamma) \times L^{2}(\Gamma)$.

PROOF. The theorem follows from the results in [7] by setting $a=0, A_{1}=A_{2}$ and $\mu=1$. In particular $H$ is bijective if and only if the two conditions $(A)$ and $(\tilde{A})$ are satisfied (see [7]). In our model problem this is easily verified. For $f \in H^{1}(\Gamma)$ and $g \in L^{2}(\Gamma)$ the right-hand side of (4) lies in $H^{1}(\Gamma) \times L^{2}(\Gamma)$ [6] such that [7, Theorem $6.1]$ yields $(v, \phi) \in H^{1}(\Gamma) \times L^{2}(\Gamma)$.

We conclude the following result which is important for the derivation of a posteriori error bounds for the boundary-element Galerkin solution of the system of integral equations (4).

COROLLARY 1. The linear operator $H$ maps $H^{1}(\Gamma) \times L^{2}(\Gamma)$ bijectively and continuously onto itself. Its inverse is also bounded, mapping $H^{1}(\Gamma) \times L^{2}(\Gamma)$ onto itself.

PROOF. From the above-mentioned properties of $V, K, K^{\prime}, W$ (proved in [6, 7]) we have that $H$ maps $H^{1}(\Gamma) \times L^{2}(\Gamma)$ continuously into itself. Let $\left(\begin{array}{l}v \\ \phi\end{array}\right) \in H^{1}(\Gamma) \times L^{2}(\Gamma)$. If $H\left(\begin{array}{l}v \\ \phi\end{array}\right)=0$ then $\left(\begin{array}{l}v \\ \phi\end{array}\right)=0$ since $H: H^{1 / 2}(\Gamma) \times H^{-1 / 2}(\Gamma) \rightarrow H^{1 / 2}(\Gamma) \times H^{-1 / 2}(\Gamma)$ is injective. Since $H: H^{1 / 2}(\Gamma) \times H^{-1 / 2}(\Gamma) \rightarrow H^{1 / 2}(\Gamma) \times H^{-1 / 2}(\Gamma)$ is bijective, given $\left(\begin{array}{l}v \\ \phi\end{array}\right) \in H^{1}(\Gamma) \times L^{2}(\Gamma)$ then some $\left(\begin{array}{l}w \\ \psi\end{array}\right) \in H^{1 / 2}(\Gamma) \times H^{-1 / 2}(\Gamma)$ with $H\left(\begin{array}{l}w \\ \psi\end{array}\right)=\left(\begin{array}{l}v \\ \phi\end{array}\right)$. Due to Theorem 1(c), $\left(\begin{array}{l}w \\ \psi\end{array}\right) \in H^{1}(\Gamma) \times L^{2}(\Gamma)$. Thus, $H$ maps $H^{1}(\Gamma) \times L^{2}(\Gamma)$ onto itself and the claimed properties of $H$ are already proved. The properties of its inverse are then included using the inverse mapping theorem. 


\section{A boundary-element method for the transmission problem}

In order to obtain quasi-optimal convergence of a Galerkin discretization of the boundary-integral equation (4), the strong ellipticity of the single-layer potential and the hypersingular operator is sufficient.

Let $\left(H_{h}^{1 / 2} \times H_{h}^{-1 / 2}: h \in I\right)$ be a family of finite-dimensional subspaces of $H^{1 / 2}(\Gamma) \times$ $H^{-1 / 2}(\Gamma)$ such that $\cup_{h \in I} H_{h}^{1 / 2} \times H_{h}^{-1 / 2}$ is dense in $H^{1 / 2}(\Gamma) \times H^{-1 / 2}(\Gamma), I \subseteq(0, \infty)$ with $0 \in \bar{I}$.

For $h \in I$ consider the following Galerkin procedure introduced and analyzed in [7]:

Find $\left(v_{h}, \phi_{h}\right) \in H_{h}^{1 / 2} \times H_{h}^{-1 / 2}$ such that

$$
\left\langle H\left(\begin{array}{l}
v_{h} \\
\phi_{h}
\end{array}\right)-\frac{1}{2}(1+H)\left(\begin{array}{l}
f \\
g
\end{array}\right),\left(\begin{array}{l}
w_{h} \\
\psi_{h}
\end{array}\right)\right\rangle_{H^{1 / 2}(\Gamma) \times H^{-1 / 2}(\Gamma)}=0
$$

for all $\left(w_{h}, \psi_{h}\right) \in H_{h}^{1 / 2} \times H_{h}^{-1 / 2}$.

Here $\langle,\rangle_{H^{1 / 2}(\Gamma) \times H^{-1 / 2}(\Gamma)}$ is defined by

$$
\left\langle\left(\begin{array}{l}
v \\
\phi
\end{array}\right),\left(\begin{array}{l}
w \\
\psi
\end{array}\right)\right\rangle_{H^{s}(\Gamma) \times H^{-s}(\Gamma)}:=\langle\phi, w\rangle+\langle\psi, v\rangle
$$

for $v, w \in H^{s}(\Gamma)$ and $\phi, \psi \in H^{-s}(\Gamma)$ where $0 \leq s \leq 1$ and $\langle$,$\rangle denotes duality$ between $H^{s}(\Gamma)$ and $H^{-s}(\Gamma)$. The following result holds which shows the quasioptimality of the Galerkin error.

THEOREM 2 ([7]). There exists $h_{0}>0$ such that for all $h \in I$ with $h<h_{0}$ the Galerkin equation (6) has a unique solution $\left(v_{h}, \phi_{h}\right)$. If $(v, \phi)$ solves the boundary-integral equation (4) then

$$
\left\|\left(\begin{array}{c}
v-v_{h} \\
\phi-\phi_{h}
\end{array}\right)\right\|_{H^{1 / 2}(\Gamma) \times H^{-1 / 2}(\Gamma)} \leq c_{0} \inf _{\left(w_{h}, \psi_{h}\right) \in H_{h}^{1 / 2}(\Gamma) \times H_{h}^{-1 / 2}(\Gamma)}\left\|\left(\begin{array}{c}
v-w_{h} \\
\phi-\psi_{h}
\end{array}\right)\right\|_{H^{1 / 2}(\Gamma) \times H^{-1 / 2}(\Gamma)}
$$

holds with $c_{0}>0$ independent of $h$.

From Theorem 2 the Galerkin solution in $H_{h}^{1 / 2}(\Gamma) \times H_{h}^{-1 / 2}(\Gamma)$ is only unique for sufficiently small $h$. This is not appropriate for an adaptive method where the mesh size $h$ may vary substantially. In the following we will show how to modify the Galerkin procedure to obtain a unique Galerkin solution for all $h$. The key is to eliminate the constant solutions; this is achieved by demanding that $v$ and $v_{h}$ have integral mean zero; for $\phi$ and $\phi_{h}$ this property holds automatically as Lemma 1 below shows. 
DEFINITION 1. Define

$$
\begin{aligned}
H_{0}^{s}(\Gamma) & :=\left\{w \in H^{s}(\Gamma):\langle 1, w\rangle=0\right\}, \\
H_{0}^{-s}(\Gamma) & :=\left\{\psi \in H^{-s}(\Gamma):\langle\psi, 1\rangle=0\right\},
\end{aligned}
$$

for $0 \leq s \leq 1$. We define $H^{s}(\Gamma)$ by (complex) interpolation [2]. Given the above family of finite-dimensional subspaces $\left(H_{h}^{1 / 2} \times H_{h}^{-1 / 2}: h \in I\right)$, let

$$
\begin{aligned}
H_{0 h}^{1 / 2} & :=H_{h}^{1 / 2} \cap H_{0}^{1 / 2}(\Gamma), \\
H_{0 h}^{-1 / 2} & :=H_{h}^{-1 / 2} \cap H_{0}^{-1 / 2}(\Gamma) .
\end{aligned}
$$

The following lemma shows that the integral of $\phi$ (which is the normal derivative of the potential $u_{1}$ on the inner side of the boundary $\Gamma$ ) around $\Gamma$ is zero and it gives the corresponding result for the Galerkin descretization $\phi_{h}$ of $\phi$.

LEMMA 1. If $(v, \phi) \in H^{1 / 2}(\Gamma) \times H^{-1 / 2}(\Gamma)$ solves the boundary-integral equation (4) and $\left(v_{h}, \phi_{h}\right) \in H_{h}^{1 / 2} \times H_{h}^{-1 / 2}$ solves its discretization (6), then

$$
\phi \in H_{0}^{-1 / 2}(\Gamma), \quad \phi_{h} \in H_{0 h}^{-1 / 2},
$$

provided $1 \in H_{h}^{1 / 2}$.

PROOF. We only give the proof for the discrete case $\phi_{h} \in H_{0}^{-1 / 2}(\Gamma), \phi \in H_{0}^{-1 / 2}(\Gamma)$ being proved analogously. Letting $w_{h}:=1$ and $\psi_{h}=0$ in (6), then from the definition of $H$

$$
\begin{aligned}
0 & =\left\langle W v_{h}, 1\right\rangle+\left\langle K^{\prime} \phi_{h}, 1\right\rangle-\frac{1}{2}\left\{\langle g, 1\rangle+\langle W f, 1\rangle+\left\langle K^{\prime} g, 1\right\rangle\right\} \\
& =\left\langle W 1, v_{h}\right\rangle+\left\langle\phi_{h}, K 1\right\rangle-\frac{1}{2}\{\langle g, 1\rangle+\langle W 1, f\rangle+\langle g, K 1\rangle\} .
\end{aligned}
$$

Note that $(1,0)$ are Cauchy data for the constant function 1 so that its Calderon projector maps $(1,0)$ onto $(1,0)$ (see $\left[7\right.$, Theorem 3.11]), that is, $\left(\begin{array}{l}1 \\ 0\end{array}\right)=H\left(\begin{array}{l}1 \\ 0\end{array}\right)$, proving $K 1=-1$ and $W 1=0$.

Using this, we get from the above that

$$
0=-\left\langle\phi_{h}, 1\right\rangle, \quad \text { whence } \quad \phi_{h} \in H_{0}^{-1 / 2}(\Gamma) .
$$

REMARK 2. In view of Lemma 1 we are led to reduce the trial functions for the tractions to $H_{0 h}^{-1 / 2}$ because the tractions belong to this space automatically.

Consider the piecewise-constant solution $u_{1}=1$ and $u_{2}=0$ of the problem (1)-(3) for $f=1$ and $g=0$. By Theorem $1 H\left(\begin{array}{l}1 \\ 0\end{array}\right)=\left(\begin{array}{l}1 \\ 0\end{array}\right)$, which yields

$$
\left\langle H\left(\begin{array}{l}
1 \\
0
\end{array}\right),\left(\begin{array}{l}
w \\
\psi
\end{array}\right)\right\rangle=0
$$


for all $w \in H^{1 / 2}(\Gamma)$ and $\psi \in H_{0}^{-1 / 2}(\Gamma)$. Consequently, the displacements of the corresponding Galerkin solutions are then determined only up to an additive constant. In order to fix this constant (as zero in the integral mean) we are led to the trial spaces $H_{0 h}^{1 / 2}(\Gamma) \times H_{o h}^{-1 / 2}(\Gamma)$.

Lemma 1 and Remark 2 motivate the following modified Galerkin procedure.

Find $\left(\bar{v}_{h}, \phi_{h}\right) \in H_{0 h}^{1 / 2} \times H_{0 h}^{-1 / 2}$ such that

$$
\left\langle H\left(\begin{array}{l}
\bar{v}_{h} \\
\phi_{h}
\end{array}\right)-\frac{1}{2}(1+H)\left(\begin{array}{l}
f \\
g
\end{array}\right),\left(\begin{array}{l}
w_{h} \\
\psi_{h}
\end{array}\right)\right\rangle_{H^{1 / 2}(\Gamma) \times H^{-1 / 2}(\Gamma)}=0
$$

for all $\left(w_{h}, \psi_{h}\right) \in H_{0 h}^{1 / 2} \times H_{0 h}^{-1 / 2}$.

The relation between the Galerkin procedures (6) and (7) are given in the following theorem.

THEOREM 3. (a) If $\left(v_{h}, \phi_{h}\right) \in H_{h}^{1 / 2} \times H_{h}^{-1 / 2}$ solves (6), then $\left(\bar{v}_{h}, \phi_{h}\right) \in H_{0 h}^{1 / 2} \times H_{0 h}^{-1 / 2}$ solves (7), where $\bar{v}_{h}:=v_{h}-v_{0 h} \in H_{0 h}^{1 / 2}$ with the constant $v_{0 h}:=\left\langle v_{h}, 1\right\rangle /\langle 1,1\rangle \in \mathbb{R}$. (b) Conversely, if $\left(\bar{v}_{h}, \phi_{h}\right) \in H_{0 h}^{1 / 2} \times H_{0 h}^{-1 / 2}$ solves (7), then $\left(v_{h}, \phi_{h}\right) \in H_{h}^{1 / 2} \times H_{h}^{-1 / 2}$ solves (6), where $v_{h}=\bar{v}_{h}+v_{0 h}$ and the constant $v_{0 h}$ is given as

$$
v_{0 h}:=\frac{\left\langle 1, \frac{1}{2}(K-1) f-\frac{1}{2} V g+V \phi_{h}-K \bar{v}_{h}\right\rangle}{\langle 1,1\rangle} \in \mathbb{R} .
$$

PROOF. If $v_{0 h} \in \mathbb{R}$ is constant and $v_{h}=\bar{v}_{h}+v_{0 h}$, then

$$
\begin{aligned}
\left\langle H\left(\begin{array}{l}
v_{h} \\
\phi_{h}
\end{array}\right)-\frac{1}{2}(1+H)\left(\begin{array}{l}
f \\
g
\end{array}\right),\left(\begin{array}{l}
w_{h} \\
\psi_{h}
\end{array}\right)\right\rangle_{H^{1 / 2}(\Gamma) \times H^{-1 / 2}(\Gamma)} \\
=\left\langle H\left(\begin{array}{l}
\bar{v}_{h} \\
\phi_{h}
\end{array}\right)-\frac{1}{2}(1+H)\left(\begin{array}{l}
f \\
g
\end{array}\right),\left(\begin{array}{l}
w_{h} \\
\psi_{h}
\end{array}\right)\right\rangle_{H^{1 / 2}(\Gamma) \times H^{-1 / 2}(\Gamma)}+v_{0 h}\left\langle 1, \psi_{h}\right\rangle
\end{aligned}
$$

for any $\left(w_{h}, \psi_{h}\right) \in H_{h}^{1 / 2} \times H_{h}^{-1 / 2}$. Hence, if $\left(v_{h}, \phi_{h}\right) \in H_{h}^{1 / 2} \times H_{h}^{-1 / 2}$ solves (6), then (7) is satisfied by $\left(\bar{v}_{h}, \phi_{h}\right)$ as well, and this proves (a), because $\psi_{h}, \phi_{h} \in H_{0 h}^{-1 / 2}$ (see Lemma 1) in (8).

Conversely, if $\left(\bar{v}_{h}, \phi_{h}\right) \in H_{0 h}^{1 / 2} \times H_{0 h}^{-1 / 2}$ solves (7), then define $v_{h}$ as in (b). Due to (8), (6) is satisfied whenever $\left(w_{h}, \psi_{h}\right) \in H_{0 h}^{1 / 2} \times H_{0 h}^{-1 / 2}$. Thus, it remains to directly check (6) for $\left(w_{h}, \psi_{h}\right)=(1,0)$ and $\left(w_{h}, \psi_{h}\right)=(0,1)$. Using $H\left({ }_{\phi}^{v}\right)=\frac{1}{2}(1+H)\left(\begin{array}{l}f \\ g\end{array}\right)$, we have in the first case

$$
\begin{aligned}
\left\langle H\left(\begin{array}{c}
v_{h}-v \\
\phi_{h}-\phi
\end{array}\right),\left(\begin{array}{l}
1 \\
0
\end{array}\right)\right\rangle_{H^{1 / 2}(\Gamma) \times H^{-1 / 2}(\Gamma)} & =\left\langle W\left(v_{h}-v\right)+K^{\prime}\left(\phi_{h}-\phi\right), 1\right\rangle \\
& =\left\langle\phi_{h}-\phi, K 1\right\rangle=0,
\end{aligned}
$$


since $K 1=-1$ and $\left\langle\phi_{h}, 1\right\rangle=0$ by the present choice of the trial functions and $\langle\phi, 1\rangle \doteq 0$ by Lemma 1 . In the second case

$$
\begin{aligned}
\left\langle H\left(\begin{array}{c}
\bar{v}_{h}+v_{0 h} \\
\phi_{h}
\end{array}\right)-\frac{1}{2}(1+H)\left(\begin{array}{l}
f \\
g
\end{array}\right),\left(\begin{array}{l}
0 \\
1
\end{array}\right)\right\rangle_{H^{1 / 2}(\Gamma) \times H^{-1 / 2}(\Gamma)} \\
\quad=\left\langle 1,-K\left(\bar{v}_{h}-v_{h 0}\right)+V \phi_{h}-\frac{1}{2}(1-K) f-\frac{1}{2} V g\right\rangle \\
\quad=v_{h 0}\langle 1,1\rangle+\left\langle 1, V \phi_{h}-K \bar{v}_{h}+\frac{1}{2}(K-1) f-\frac{1}{2} V g\right\rangle
\end{aligned}
$$

which is zero due to the definition of $v_{h 0}$. Altogether, (6) is satisfied for all $\left(w_{h}, \psi_{h}\right) \in$ $H_{h}^{1 / 2} \times H_{h}^{-1 / 2}$ which proves (b).

The proof of the following lemma can be concluded from $[13,19,16]$.

LEMMA 2. The single-layer potential $V$ is positive definite on $H_{0}^{-1 / 2}(\Gamma)$ and the hypersingular operator $W$ is positive definite on $H_{0}^{1 / 2}(\Gamma)$, that is, there exists some constant $c>0$ such that

$$
\langle\psi, V \psi\rangle \geq c \cdot\|\psi\|_{H^{-1 / 2}(\Gamma)}^{2}, \quad\langle W w, w\rangle \geq c \cdot\|w\|_{H^{1 / 2}(\Gamma)}^{2}
$$

for all $w \in H_{0}^{1 / 2}(\Gamma)$ and all $\psi \in H_{0}^{-1 / 2}(\Gamma)$.

As an application of Lemma 2 we obtain the quasi-optimality of the modified Galerkin procedure (7).

THEOREM 4. There exists $c_{0}>0$ such that for all $h \in I$ the (modified) Galerkin equation (7) has a unique solution $\left(\bar{v}_{h}, \phi_{h}\right)$ and

$$
\left\|\left(\begin{array}{c}
\bar{v}-\bar{v}_{h} \\
\phi-\phi_{h}
\end{array}\right)\right\|_{H^{1 / 2}(\Gamma) \times H^{-1 / 2}(\Gamma)} \leq c_{0} \inf _{\left(w_{h}, \psi_{h}\right) \in H_{0 h}^{1 / 2} \times H_{0 h}^{-1 / 2}}\left\|\left(\begin{array}{c}
v-w_{h} \\
\phi-\psi_{h}
\end{array}\right)\right\|_{H^{1 / 2}(\Gamma) \times H^{-1 / 2}(\Gamma)}
$$

holds, where $(v, \phi)$ is the solution of (4) and $\bar{v}:=v-\langle 1, v\rangle /\langle 1,1\rangle \in H_{0}^{1 / 2}(\Gamma)$.

PROOF. Note that, due to Lemma 2, the bilinear form

$$
\langle H \cdot, \cdot\rangle_{H^{1 / 2}(\Gamma) \times H^{-1 / 2}(\Gamma)}:\left(H_{0}^{1 / 2}(\Gamma) \times H_{0}^{-1 / 2}(\Gamma)\right)^{2} \rightarrow \mathbb{R}
$$

is (symmetric and) positive definite. For all $(v, \phi) \in H_{0}^{1 / 2}(\Gamma) \times H_{0}^{-1 / 2}(\Gamma)$,

$$
\left\langle H\left(\begin{array}{l}
v \\
\phi
\end{array}\right),\left(\begin{array}{l}
v \\
\phi
\end{array}\right)\right\rangle=\langle W v, v\rangle+\langle V \phi, \phi\rangle \geq c \cdot\left\|\left(\begin{array}{l}
v \\
\phi
\end{array}\right)\right\|_{H_{0}^{1 / 2}(\Gamma) \times H_{0}^{-1 / 2}(\Gamma)} .
$$

The proof is concluded by standard arguments. 
REMARK 3. Combining Theorem 4 with Theorem 3 we get in Theorem 2 that $h_{0}=\infty$, that is, there is no restriction on $h$ in order to guaranteee a unique solution of the Galerkin procedure and the error estimate.

\section{An a posteriori error estimate}

In order to state the a posteriori estimate for the h-version of the boundary-element method (7) we need some notation about the mesh used, that is, on the discrete function spaces.

DEFINITION 2. Let $\Gamma=\partial \Omega$ be a polygonal boundary. Given $k>0, c_{k}>0$ and integers $p_{1} \geq 1$ and $p_{0} \geq 0$, the class $\Sigma_{k, c_{k}, p_{1}, p_{0}}$ is the set of all triples $\left(T, S_{h}^{0}, S_{h}^{1}\right)$ where

(a1) $T=\left\{\Gamma_{i}: i=1, \ldots, n_{T}\right\}$ is a set of interval domains (that is, straight lines) of $\Gamma$ which partition $\Gamma$. Let $h_{j}>0$ be the Euclidean length of the element $\Gamma_{j}, j=$ $1, \ldots, n_{T}$. Define $\Gamma_{n_{T}+j}=\Gamma_{j}$ and $h_{n_{T}+j}=h_{j}$ for any integer $j$, that is, use the indices modulo $n_{T}$. Then, assume that for any integers $i, j$

$$
\frac{h_{i}}{h_{j}} \leq c_{k} \cdot k^{|i-j|}
$$

holds.

(a2) $S_{h}^{1}$ and $S_{h}^{0}$ are piecewise polynomials of degree $p_{1}$ and $p_{0}$, respectively, that is,

$$
\begin{aligned}
& S_{h}^{1}:=\left\{\eta \in C(\Gamma):\left.\eta\right|_{\Gamma_{j}} \text { is a polynomial of degree } \leq p_{1}, j=1, \ldots, n_{T}\right\} \\
& S_{h}^{0}:=\left\{\eta \in L^{\infty}(\Gamma):\left.\eta\right|_{\Gamma_{j}} \text { is a polynomial of degree } \leq p_{0}, j=1, \ldots, n_{T}\right\} .
\end{aligned}
$$

Given $\left(T, S_{h}^{0}, S_{h}^{1}\right) \in \Sigma_{k, c_{k}, p_{1}, p_{0}}$ let

$$
P_{h}^{j}: L^{2}(\Gamma) \rightarrow S_{h}^{j}, \quad j=0,1,
$$

be the $L^{2}$ projection onto $S_{h}^{j}$ and let $h(T) \in L^{\infty}(\Gamma)$ be piecewise constant with the value of the element length, that is, $\left.h(T)\right|_{\Gamma_{j}}:=h_{j}, j=1, \ldots, n_{T}$. With $S_{h}^{1}$ and $S_{h}^{0}$ we associate the trial spaces $H_{0 h}^{1 / 2}:=H_{0}^{1 / 2}(\Gamma) \cap S_{h}^{1}$ and $H_{0 h}^{-1 / 2}:=H_{0}^{-1 / 2}(\Gamma) \cap S_{h}^{0}$.

REMARK 4. Note that quasi-uniform meshes are included in $\Sigma_{k, c_{k}, p_{1}, p_{0}}$ for which it is well known that $P_{h}^{1}$ is stable in $L^{2}$ and $H^{1}$, that is, the mappings

$$
P_{h}^{1}: L^{2}(\Gamma) \rightarrow L^{2}(\Gamma) \quad \text { and } \quad P_{h}^{1}: H^{1}(\Gamma) \rightarrow H^{1}(\Gamma)
$$

are continuous. It is proved, for example in [9], for the one-dimensional case that this holds for meshes with (9) as well. Compare [9, Theorem 2] and the references therein 
for former results. In our one-dimensional case (without homogeneous but with periodic boundary conditions) the stability of the $L^{2}$-projection follows immediately from that result.

For the above-mentioned meshes we have the following a posteriori estimate.

THEOREM 5. Let $(v, \phi) \in H_{0}^{1}(\Gamma) \times L^{2}(\Gamma)$ solve the boundary integral equation (4) and let $\bar{v}:=v-\langle 1, v\rangle /\langle 1,1\rangle$. Let $\left(\bar{v}_{h}, \phi_{h}\right) \in H_{0 h}^{1 / 2} \times H_{0 h}^{-1 / 2}$ solve the discrete boundary integral equation (7), where $H_{0 h}^{1 / 2}:=H_{0 h}^{1 / 2}(\Gamma) \cap S_{h}^{1}, \quad H_{0 h}^{-1 / 2}:=H_{0 h}^{-1 / 2}(\Gamma) \cap S_{h}^{0}$ and $\left(T, S_{h}^{0}, S_{h}^{1}\right) \in \Sigma_{k, c_{k}, p_{1}, p_{0}}$.

Then, there exists a constant $c>0$ depending only on $k, c_{k}, p_{1}, p_{0}$ with

$$
\begin{aligned}
\| \bar{v}- & \bar{v}_{h}\left\|_{H^{1 / 2}(\Gamma)}^{2}+\right\| \phi-\phi_{h} \|_{H^{1 / 2}(\Gamma)}^{2} \\
& \leq c\left(\left\|h(T) \cdot R_{1}^{\prime}\right\|_{L^{2}(\Gamma)}\left\|R_{1}^{\prime}\right\|_{L^{2}(\Gamma)}+\left\|h(T) \cdot R_{2}\right\|_{L^{2}(\Gamma)}\left\|R_{2}\right\|_{L^{2}(\Gamma)}\right),
\end{aligned}
$$

where

$$
\begin{aligned}
& R_{1}:=\frac{1}{2}(f-K f+V g)-V \phi_{h}+K v_{h}, \\
& R_{2}:=\frac{1}{2}\left(g+K^{\prime} g+W f\right)-W v_{h}-K^{\prime} \phi_{h} .
\end{aligned}
$$

\section{Proof of the a posteriori estimate}

The proof of Theorem 5 is related to Lemma 3 and Lemma 5.

LEMMA 3. There exists a constant $c>0$, depending on $\Sigma_{k, c_{k}, p_{1}, p_{0}}$, such that for all $\left(T, S_{h}^{0}, S_{h}^{1}\right) \in \Sigma_{k, c_{k}, p_{1}, p_{0}}, f \in H_{0}^{0}(\Gamma)$ and $g \in H^{1}(\Gamma)$

$$
\left\langle f, g-P_{h}^{1} g\right\rangle \leq c\|g\|_{H^{1 / 2}(\Gamma)} \sqrt{\|f\|_{L^{2}(\Gamma)} \cdot\|h(T) \cdot f\|_{L^{2}(\Gamma)}} .
$$

PROOF. We start proving that the continuous function $e:=g-g_{h}, g_{h}:=P_{h}^{1} g$ has at least one zero at the closure of $\Gamma_{j} \cup \Gamma_{j+1}$ for any $j=1, \ldots, n$ (recall that we use the lower indices here modulo $n:=n_{T}$ ). Assume that this is false, that is, assume that $e$ is positive, say, on $\Gamma_{j} \cup \Gamma_{j+1}$. Since $e$ is continuous we easily construct a nonnegative function $\eta \in S_{h}^{1}$ (for example, piecewise linear), $\eta \neq 0$, with support in the closure of $\Gamma_{j} \cup \Gamma_{j+1}$ such that $e-\eta$ is still positive on $\Gamma_{j} \cup \Gamma_{j+1}$. Then we have that

$$
\int_{\Gamma}|e-\eta|^{2} d s<\int_{\Gamma}|e|^{2} d s
$$

which contradicts $g_{h}$ ( and not $g_{h}-\eta$ ) being the $L^{2}$-projection of $g$ onto $S_{h}^{1}$. 
Hence we may define a second partition $\tilde{\Gamma}_{1}, \ldots, \tilde{\Gamma}_{m}$ of $\Gamma$ (not necessarily straight lines, but at least a union of two straight lines) such that the endpoint of $\tilde{\Gamma}_{j}$ is the beginning of $\tilde{\Gamma}_{j+1}$ and is a zero of $e$. We may assume that $m$ is chosen maximal such that $\tilde{\Gamma}_{j}$ has positive length. Then, we have proved above that the length of $\tilde{\Gamma}_{j}$ is smaller than $c_{1} h_{k(j)}$ where $k(j) \in\left\{1, \ldots, n_{T}\right\}$ is fixed with minimal $h_{k}$ and $\tilde{\Gamma}_{j} \cap \Gamma_{k} \neq \emptyset$, that is,

$$
h_{k(j)}=\min \left\{h_{k}: \Gamma_{k} \cap \tilde{\Gamma}_{j} \neq \emptyset\right\} .
$$

Note that $c_{1}$ depends only on $k$ and $c_{k}$ (see (9)). Note also that $\int_{\tilde{\Gamma}_{j}} e^{\prime} d s=0$ due to the construction of the partition $\tilde{\Gamma}_{1}, \ldots, \tilde{\Gamma}_{m}$.

Since $\langle f, 1\rangle=0$ we may define $F \in H^{1}(\Gamma)$ by piecewise integration of $f$ along $\Gamma$. Note that, if we start with $F\left(x_{0}\right)=: c_{0}$ at $x_{0} \in \Gamma_{1}$, then $F$ is continuous and we can determine $c_{0}$ uniquely such that $F \in H_{0}^{1}(\Gamma)$ and $F^{\prime}=f$.

Next we construct a simple function $\eta \in L^{2}(\Gamma)$ which is constant on any $\tilde{\Gamma}_{j}$ with the value of the continuous function $F$ at the beginning of $\tilde{\Gamma}_{j}$. Hence, by the fundamental theorem of calculus,

$$
\|F-\eta\|_{L^{\infty}\left(\tilde{\Gamma}_{j}\right)} \leq\|f\|_{L^{1}\left(\tilde{\Gamma}_{j}\right)} \leq \sqrt{c_{1} h_{k(j)}}\|f\|_{L^{2}\left(\tilde{\Gamma}_{j}\right)} .
$$

Due to $\int_{\tilde{\Gamma}_{j}} e^{\prime} d s=0$ we have $\int_{\Gamma} e^{\prime} \cdot \eta d s=0$. Using this and integration by parts we have

$$
\begin{aligned}
\langle f, e\rangle & =\left\langle F^{\prime}, e\right\rangle=-\left\langle F, e^{\prime}\right\rangle=\left\langle\eta-F, e^{\prime}\right\rangle \\
& =\sum_{j=1}^{m} \int_{\tilde{\Gamma}_{j}}(\eta-F) e^{\prime} d s \\
& \leq \sum_{j=1}^{m} c_{1} h_{k(j)}\|f\|_{L^{2}\left(\tilde{\Gamma}_{j}\right)}\left\|e^{\prime}\right\|_{L^{2}\left(\tilde{\Gamma}_{j}\right)} \\
& \leq c_{1}\left\|e^{\prime}\right\|_{L^{2}(\Gamma)} \sqrt{\sum_{j=1}^{m} h_{k(j)}^{2}\|f\|_{L^{2}\left(\tilde{\Gamma}_{j}\right)}^{2}}
\end{aligned}
$$

by Cauchy's inequality. Next we define $h:=h(T)$ which is constant with value $h_{j}$ on $\Gamma_{j}$ and $\tilde{h}$ constant with value $h_{k(j)}$ on $\tilde{\Gamma}_{j}$. By the construction of $k(j)$ we have $h \geq \tilde{h}$ a.e. on $\Gamma$. Thus

$$
\sum_{j=1}^{m} h_{k(j)}^{2}\|f\|_{L^{2}\left(\tilde{\Gamma}_{j}\right)}^{2}=\|\tilde{h} f\|_{L^{2}(\Gamma)}^{2} \leq\|h f\|_{L^{2}(\Gamma)}^{2}
$$

which leads to

$$
\left\langle f, g-g_{h}\right\rangle \leq c_{1}\left\|g^{\prime}-g_{h}^{\prime}\right\|_{L^{2}(\Gamma)}\|h(T) \cdot f\|_{L^{2}(\Gamma)} .
$$


Because of this and Cauchy's inequality, we conclude from the stability of $P_{h}^{1}$ (compare Remark 4) that, for fixed $f \in H_{0}^{0}(\Gamma)$, the linear mapping

$$
T_{f}: H^{s}(\Gamma) \rightarrow \mathbb{R}, \quad g \mapsto\left\langle f, g-P_{h}^{1} g\right\rangle
$$

is bounded for $s=0,1$. Interpolating the corresponding bounds gives the bound

$$
c_{2} \sqrt{\|f\|_{L^{2}\left(\Gamma_{j}\right)}\|h(T) \cdot f\|_{L^{2}\left(\Gamma_{j}\right)}}
$$

for the norm of $T_{f}$ in the case $s=1 / 2$ [2]. Note that $c_{2}$ depends on $c_{1}$ and on the stability constants of the $L^{2}$-projections, that is, on $k, c_{k}, p_{0}, p_{1}$. This proves the lemma.

We need also an analog of Lemma 3 where the projection works in the first component, that is, a projection on $S_{h}^{0}$ acting in $H^{-1}(\Gamma)$. In order to do this we need further notation.

LEMMA 4. After changing to an equivalent norm in $H_{0}^{-1}(\Gamma)$, there exists an isomorphism $I: H_{0}^{-1}(\Gamma) \rightarrow L^{2}(\Gamma)$ with

$$
(I(\phi))^{\prime}=\phi
$$

for all $\phi \in L^{2}(\Gamma)$, where prime denotes differentiation. In other words, differentiation is an isomorphism between $L^{2}(\Gamma)$ and $H_{0}^{-1}(\Gamma)$ with an inverse 1 .

PROOF. Let $I: H_{0}^{0}(\Gamma) \rightarrow H_{0}^{1}(\Gamma)$ denote the integration used in the proof of Lemma 3 , that is, for all $\phi \in H_{0}^{0}(\Gamma),(I(\phi))^{\prime}=\phi$ and $I(\phi) \in H_{0}^{1}(\Gamma)$ holds. It is easily seen that $I: H_{0}^{0}(\Gamma) \rightarrow H_{0}^{1}(\Gamma)$ is linear and bounded if we consider the $H^{-1}$-norm in $H_{0}^{0}(\Gamma)$ and $L^{2}$-norm in $H_{0}^{1}(\Gamma)$. Hence we may extend $I$ uniquely to a bounded linear mapping

$$
I: H_{0}^{-1} \rightarrow H_{0}^{0}(\Omega) .
$$

It is not hard to see that $I$ is the inverse of differentiation and hence $I$ is an isomorphism if we endow $H_{0}^{-1}(\Gamma)$ with the equivalent norm

$$
\|\phi\|_{H_{0}^{-1}(\Gamma)}:=\|I \phi\|_{L^{2}(\Gamma)}, \quad \phi \in H_{0}^{-1}(\Gamma) .
$$

DEFINITION 3. Given $\left(T, S_{h}^{0}, S_{h}^{1}\right) \in \Sigma_{k, c_{k}, p_{1}, p_{0}}$ let $\tilde{P}_{h}^{1}$ be the $L^{2}$-projection on

$$
\tilde{S}_{h}^{1}:=\left\{\eta \in C(\Gamma):\left.\eta\right|_{\Gamma_{j}} \text { is a polynomial of degree } \leq p_{0}+1, j=1, \ldots, n_{T}\right\} .
$$

We define $\tilde{P}_{h}^{0}: H_{0}^{-1}(\Gamma) \rightarrow S_{h}^{0}$ by

$$
\tilde{P}_{h}^{0}:=I^{-1} \circ \tilde{P}_{h}^{1} \circ I .
$$


REMARK 5. Note that $\tilde{P}_{h}^{0}: H_{0}^{-1}(\Gamma) \rightarrow S_{h}^{0}$ is a projection and, according to the stability of $P_{h}^{1}$ (see Remark 4), we have that the norm of $\tilde{P}_{h}^{0}: H_{0}^{-s}(\Gamma) \rightarrow H_{0}^{-s}(\Gamma)$ is bounded by a constant which depends on $\Sigma_{k, c_{k}, p_{1}, p_{0}}$ for $s=0,1$.

LEMMA 5. There exists a constant $c>0$ depending on $\Sigma_{k, c_{k}, p_{1}, p_{0}}$ such that for all $f \in H_{0}^{0}(\Gamma)$ and $g \in H^{1}(\Gamma)$

$$
\left\langle f-\tilde{P}_{h}^{0} f, g\right\rangle \leq c\|f\|_{H^{-1 / 2}(\Gamma)} \sqrt{\left\|g^{\prime}\right\|_{L^{2}(\Gamma)} \cdot\left\|h(T) \cdot g^{\prime}\right\|_{L^{2}(\Gamma)}} .
$$

ProOF. The proof is quite similar to the proof of Lemma 3 and is outlined using the above notation.

Let $f_{h}:=\tilde{P}_{h}^{0} f$ and $e:=I f-I f_{h}=I f-\tilde{P}_{h}^{1} I f_{h}$. As in the proof of Lemma 3 we conclude that the continuous functions $e$ has some zeros which leads to a partition $\tilde{\Gamma}_{1}, \ldots, \tilde{\Gamma}_{m}$ of $\Gamma$. Concerning the values of the continuous function $g$ we find a function $\eta$ constant on each $\tilde{\Gamma}_{j}$ with $\left\langle f-f_{h}, g\right\rangle=\left\langle f-f_{h}, g-\eta\right\rangle$ and

$$
\|g-\eta\|_{L^{\infty}\left(\tilde{\Gamma}_{j}\right)} \leq \sqrt{c_{1} h_{k(j)}}\left\|g^{\prime}\right\|_{L^{2}\left(\tilde{\Gamma}_{j}\right)} .
$$

Arguing as in the proof of Lemma 3 we then conclude that

$$
\left\langle f-f_{h}, g\right\rangle \leq c_{2}\left\|f-f_{h}\right\|_{L^{2}(\Gamma)}\left\|h(T) \cdot g^{\prime}\right\|_{L^{2}(\Gamma)} .
$$

Integration by parts shows that

$$
\left\langle f-f_{h}, g\right\rangle=-\left\langle e, g^{\prime}\right\rangle \leq\left\|f-f_{h}\right\|_{H_{0}^{-1}(\Gamma)}\left\|g^{\prime}\right\|_{L^{2}(\Gamma)},
$$

since $I$ is an isomorphism.

Thus, using the stability of $\tilde{P}_{h}^{0}$ (see Remark 5) we have proved that, for fixed $g \in H^{1}(\Gamma)$, the linear mapping

$$
T_{g}: H_{0}^{-s}(\Gamma) \rightarrow \mathbb{R}, \quad f \mapsto\left\langle f-\tilde{P}_{h}^{0} f, g\right\rangle
$$

is bounded for $s=0,1$. Interpolating the corresponding bounds yields the bound

$$
c_{3} \sqrt{\left\|g^{\prime}\right\|_{L^{2}(\Gamma)}\left\|h(T) \cdot g^{\prime}\right\|_{L^{2}(\Gamma)}}
$$

for the norm of $T_{g}$ in the case $s=1 / 2[2]$.

PRoof OF THEOREM 5. Define $d:=v-v_{h} \in H^{1}(\Gamma), \bar{d}:=\bar{v}-\bar{v}_{h} \in H_{0}^{1}(\Gamma)$ and $e:=\phi-\phi_{h} \in H_{0}^{0}(\Gamma)$. According to Lemma 2 we have a constant $c_{1}>0$ such that 
(recall $W 1=0$ )

$$
\begin{aligned}
\frac{1}{c_{1}}\left(\|\bar{d}\|_{H^{1 / 2}(\Gamma)}^{2}+\|e\|_{H^{-1 / 2}(\Gamma)}^{2}\right) & \leq\langle e, V e\rangle+\langle W \bar{d}, \bar{d}\rangle \\
& =\langle e, V e\rangle+\langle W d, d\rangle=\left\langle H\left(\begin{array}{l}
d \\
e
\end{array}\right),\left(\begin{array}{l}
d \\
e
\end{array}\right)\right\rangle \\
& =\left\langle H\left(\begin{array}{l}
d \\
e
\end{array}\right),\left(\begin{array}{l}
d-d_{h} \\
e-e_{h}
\end{array}\right)\right\rangle,
\end{aligned}
$$

where the projection property of the Galerkin procedure is used for arbitrary $d_{h} \in H_{h}^{1 / 2}$ and $e_{h} \in H_{h}^{-1 / 2}$. According to the definition of $H$ we have

$$
\begin{aligned}
\frac{1}{c_{1}}\left(\|\bar{d}\|_{H^{1 / 2}(\Gamma)}^{2}+\|e\|_{H^{-1 / 2}(\Gamma)}^{2}\right) & \leq\left\langle H\left(\begin{array}{l}
d \\
e
\end{array}\right),\left(\begin{array}{c}
d-d_{h} \\
e-e_{h}
\end{array}\right)\right\rangle \\
& =\left\langle e-e_{h}, V e-K d\right\rangle+\left\langle W d+K^{\prime} e, d-d_{h}\right\rangle \\
& =\left\langle e-e_{h}, V e-K d\right\rangle+\left\langle W d+K^{\prime} e, \bar{d}-d_{h}\right\rangle .
\end{aligned}
$$

We may choose $d_{h}:=P_{h}^{1} \bar{d}$ and $e_{h}:=\tilde{P}_{h}^{0} e$ as in Lemma 3 and Lemma 5, which concludes the proof since

$$
R_{1}=V e-K d, \quad R_{2}=W d+K^{\prime} e \in H_{0}^{0}(\Gamma) .
$$

\section{The adaptive procedure}

In this section the class $\Sigma:=\Sigma_{k, c_{k}, p_{0}, p_{1}}$ of discretizations $\left(T, S_{h}^{0}, S_{h}^{1}\right)$ of $\Gamma$ is considered and the adaptive procedure explained. Throughout this section we fix $k, c_{k}, p_{0}, p_{1}$, writing $\Sigma$ instead of $\Sigma_{k, c_{k}, p_{0}, p_{1}}$.

REMARK 6. Given TOL $>0$, we cannot look for $\left(T, S_{h}^{0}, S_{h}^{1}\right) \in \Sigma$ such that

$$
\left\|\bar{v}-\bar{v}_{h}\right\|_{H^{1 / 2}(\Gamma)}+\left\|\phi-\phi_{h}\right\|_{H^{-1 / 2}(\Gamma)} \leq \mathrm{TOL}
$$

because in general we do not know $(v, \phi)$. Instead Theorem 5 leads us to look for $\left(T, S_{h}^{0}, S_{h}^{1}\right) \in \Sigma$ such that

$$
\left\|h(T) \cdot R_{1}^{\prime}\right\|_{L^{2}(\Gamma)}\left\|R_{1}^{\prime}\right\|_{L^{2}(\Gamma)}+\left\|h(T) \cdot R_{2}\right\|_{L^{2}(\Gamma)}\left\|R_{2}\right\|_{L^{2}(\Gamma)} \leq \mathrm{TOL}^{2}
$$

which guarantees (11) up to a multiplicative constant $c>0$. Since the constant $c$ in the a posteriori estimate in Theorem 5 is unknown we cannot compute an error estimate. Hence, the goal of this contribution is only to hint how to obtain good meshes in practice. 
We are concerned with the following problem in order to steer the mesh refinement.

DEFINITION 4. Given TOL $>0$ find $\left(T, S_{h}^{0}, S_{h}^{1}\right) \in \Sigma$ such that $n_{T}$ is minimal and the related Galerkin solution $\left(\bar{v}_{h}, \phi_{h}\right)$ of (7) satisfies

$$
\left\|h(T) R_{1}^{\prime}\right\|_{L^{2}(\Gamma)}\left\|R_{1}^{\prime}\right\|_{L^{2}(\Gamma)}+\left\|h(T) R_{2}\right\|_{L^{2}(\Gamma)}\left\|R_{2}\right\|_{L^{2}(\Gamma)} \leq \mathrm{TOL}^{2},
$$

where $R_{1}, R_{2}$ are defined as in Theorem 5. If such optimal $\left(T, S_{h}^{0}, S_{h}^{1}\right) \in \Sigma$ exists it is called an efficient discretization (with respect to the error estimator of Theorem 5).

REMARK 7. The notion of efficient meshes is to compare two discretizations satisfying the estimate (12) with the same TOL. In view of Theorem 5 the same guaranteed error estimate is obtained with the a posteriori error $c$. TOL. Clearly, $c$. TOL is unknown, but our goal is to compare the two meshes in view of computational costs for the related Galerkin solutions. That is, to compare the related number of degrees of freedom (for simplicity) of two meshes which yield the same guaranteed (but unknown) error $c$. TOL. Hence, a mesh is optimal (with respect to the error estimator of Theorem 5) if it is related to an efficient discretization in the sense of Definition 4.

The following theorem shows that the adaptive discretization method based on (12) is operative in the sense of $[11,14]$ (and the work of Eriksson and Johnson quoted there).

THEOREM 6. For any TOL we can find $\left(T, S_{h}^{0}, S_{h}^{1}\right) \in \Sigma$ such that the related Galerkin solution $\left(\bar{v}_{h}, \phi_{h}\right)$ of (7) satisfies (12), where $R_{1}, R_{2}$ are defined as in Theorem 5.

Proof. Because of Theorem 1 for quasi-uniform meshes we get from [7, p.407] that

$$
\left\|\left(\begin{array}{l}
\bar{v} \\
\phi
\end{array}\right)-\left(\begin{array}{l}
\bar{v}_{h} \\
\phi_{h}
\end{array}\right)\right\|_{H^{\mathrm{I}}(\Gamma) \times L^{2}(\Gamma)} \leq C \cdot\left\|\left(\begin{array}{l}
\bar{v} \\
\phi
\end{array}\right)\right\|_{H^{\prime}(\Gamma) \times L^{2}(\Gamma)},
$$

that is, stability of the Galerkin projector in the space $H^{1}(\Gamma) \times L^{2}(\Gamma)$. Consequently, $\left(\bar{v}_{h}, \phi_{h}\right)$ is bounded in $H^{1}(\Gamma) \times L^{2}(\Gamma)$ uniformly in $h$ so that $\left(R_{1}, R_{2}\right)$ are uniformly bounded in $H^{1}(\Gamma) \times L^{2}(\Gamma)$. Hence, the left-hand side of (12) tends towards zero if $\|h\|_{L^{\infty}(\Gamma)}$ tends towards zero.

It remains to show how the minimization problem (4) can be solved, at least approximately. We follow the procedure as introduced in the finite-element case by Eriksson and Johnson (see the literature in [14]) and obtain the following scheme for the boundary-element case.

Adaptive procedure. Given TOL $>0$ and $k, c_{k}, p_{0}, p_{1}$, start with a quasiuniform mesh where $c_{k}$ bounds the quotient of all mesh-sizes and $k>1$. Given $\left(T, S_{h}^{0}, S_{h}^{1}\right) \in \Sigma$ we compute some new mesh $\left(T, S_{h}^{0}, S_{h}^{1}\right) \in \Sigma$ as follows: 
(1) Compute the Galerkin solution of the integral equation (7) with respect to the discrete spaces $H_{0 h}^{1 / 2}:=H_{0}^{1 / 2}(\Gamma) \cap S_{h}^{1}$ and $H_{0 h}^{-1 / 2}:=H_{0}^{-1 / 2}(\Gamma) \cap S_{h}^{0}$ using some standard boundary-element program.

(2) Use some postprocessing to compute, at least approximately, the residues $R_{1}$ and $R_{2}$ (as explained in detail in Section 7). Thereby, for any element $\Gamma_{j} \in T$, obtain the summands

$$
\left\|R_{1}^{\prime}\right\|_{L^{2}\left(\Gamma_{j}\right)}, \quad\left\|R_{2}\right\|_{L^{2}\left(\Gamma_{j}\right)}
$$

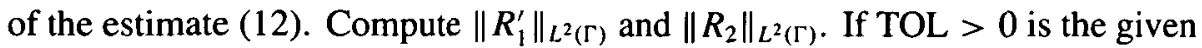
tolerance (such that the unknown value $c$. TOL should be the prescribed error) choose $\bar{h}_{j}>0$ maximal such that $\bar{h}_{j} / h_{j}$ is a natural number and

$$
\sqrt{\bar{h}_{j}}\left(\sqrt{\left\|R_{1}^{\prime}\right\|_{L^{2}\left(\Gamma_{j}\right)}\left\|R_{1}^{\prime}\right\|_{L^{2}(\Gamma)}}+\sqrt{\left\|R_{2}\right\|_{L^{2}\left(\Gamma_{j}\right)}\left\|R_{2}\right\|_{L^{2}(\Gamma)}}\right) \leq \mathrm{TOL} / n_{T} .
$$

If $\bar{h}_{j}=h_{j}$ for all $j=1, \ldots, n_{T}$ we stop the process or change TOL.

(3) Given $\bar{h}_{j}$ we construct a new triangulation $\hat{T}$ of $\Gamma$ such that we keep all knots and possibly refine the mesh on some element $\Gamma_{j}$ if $\bar{h}_{j}<h_{j}$. This gives a new partition $\bar{T}$ of $\Gamma$.

(4) Check (9) and refine the mesh again such that (9) is satisfied. For simplicity, assume $c_{k}=1, k>1$ and check all the neighbor elements in $\bar{T}$ in order that they satisfy

$$
\frac{\bar{h}_{j}}{\bar{h}_{j+1}}, \frac{\bar{h}_{j+1}}{\bar{h}_{j}} \leq k \quad\left(j=1, \ldots, n_{\bar{T}}\right) .
$$

If $\bar{h}_{j} / \bar{h}_{j+1}>k\left(\bar{h}_{j+1} / \bar{h}_{j}>k\right)$ then refine $\bar{\Gamma}_{j}\left(\bar{\Gamma}_{j+1}\right)$.

(5) If one has found a new mesh which satisfies (9) then this defines $\hat{T}$. Restart with (1).

\section{Numerical example}

We present numerical results for the solution of the integral equation (4) using the h-version of the Galerkin method (7). In all cases we take, as the simplest case, continuous piecewise-linear constant trial functions corresponding to $p_{0}=0$ and $p_{1}=1$. We start with some remarks on the numerical realization. As in $[3,4]$ we omit the restrictive condition (9) on the mesh and run a modified adaptive procedure, namely the algorithm (A4) and compare with further modifications (A1)-(A3).

7.1. The discrete problem As explained in $[12,17]$, the inner integral of the singlelayer potential and the inner integral of the adjoint of the double-layer potential can 
be computed explicitly for piecewise polynomials. Hence, the stiffness matrix of the single-layer potential operator $V$ with a typical entry

$$
\int_{\Gamma_{k}} \int_{\Gamma_{j}} \log |x-y| d s_{y} d s_{x}
$$

can be computed using 32-point Gaussian quadrature over $\Gamma_{k}$ which requires 32 evaluations of $\int_{\Gamma_{j}} \log |x-y| d s_{y}$. Similarly, the inner integral of the adjoint of the double-layer potential $K^{\prime}$ can be implemented explicitly so that the stiffness matrix of $K^{\prime}$ (which is the transpose of $K$ ) can be computed numerically by using a 32 point Gaussian quadrature rule for the outer integration. Using $W=-\frac{\partial}{\partial s} V \frac{\partial}{\partial s}[16]$ the entries of the stiffness matrix of the hypersingular integral operator can be reduced to the terms appearing in the integration of the stiffness matrix of the single-layer potential. Thus, $\left\langle W \eta_{i}, \eta_{j}\right\rangle=\left\langle V \eta_{i}^{\prime}, \eta_{j}^{\prime}\right\rangle$ for piecewise-constant functions $\eta_{i}^{\prime}$ and $\eta_{j}^{\prime}$.

The right-hand sides of the integral equations appearing in the previous sections are computed with 32-point Gaussian quadrature formulas.

The resulting linear systems of Galerkin equations are solved by a direct Gaussian elimination solver.

7.2. Energy norms and residuals Because of Lemma 2 we compute the "energy" norm,

$$
\|\psi\|_{V}:=\sqrt{\langle V \psi, \psi\rangle} \quad\left(\psi \in H^{-1 / 2}(\Gamma)\right),
$$

is computed, which is an equivalent norm on $H^{-1 / 2}(\Gamma)$. Also, we compute the energy norm

$$
\|v\|_{W}:=\sqrt{\langle W v, v\rangle} \quad\left(v \in H^{1 / 2}(\Gamma)\right)
$$

which is an equivalent norm on $H_{0}^{1 / 2}(\Gamma)$. Then we combine both equivalent norms and consider the norm $\|v\|_{W}+\|\psi\|_{v}$ for $(v, \psi) \in H_{0}^{1 / 2}(\Gamma) \times H^{-1 / 2}(\Gamma)$ being equivalent to that in $H_{0}^{1 / 2}(\Gamma) \times H^{-1 / 2}(\Gamma)$. If

$$
\bar{v}:=v-\frac{\langle 1, v\rangle}{\langle 1,1\rangle} \cdot 1 \quad \text { and } \quad \bar{v}_{h}:=v_{h}-\frac{\left\langle 1, v_{h}\right\rangle}{\langle 1,1\rangle} \cdot 1
$$

then $\left\|\left(\bar{v}-\bar{v}_{h}, \phi-\phi_{h}\right)\right\|_{H^{1 / 2}(\Gamma) \times H^{-1 / 2}(\Gamma)}^{2}$ is equivalent to

$$
\begin{gathered}
\left\langle V\left(\phi-\phi_{h}\right),\left(\phi-\phi_{h}\right)\right\rangle+\left\langle W\left(v-v_{h}\right),\left(v-v_{h}\right)\right\rangle \\
\quad=\left\langle H\left(\begin{array}{c}
v-v_{h} \\
\phi-\phi_{h}
\end{array}\right),\left(\begin{array}{c}
v-v_{h} \\
\phi-\phi_{h}
\end{array}\right)\right\rangle \\
=\left\langle\phi-\phi_{h}, R_{1}\right\rangle+\left(v-v_{h}, R_{2}\right\rangle,
\end{gathered}
$$

where $R_{1}, R_{2}$ are the residuals defined in the previous section. Given $f, g$ and knowing $v, \phi$ the $L^{2}(\Gamma)$ scalar products of $\left(\phi-\phi_{h}\right)$ with $f, V \phi_{h}, K f, K v_{h}$, and $V g$ and $\left(v-v_{h}\right)$ 
with $g, K^{\prime} g, W f, W v_{h}$, and $K^{\prime} \phi_{h}$ are computed with 32-point Gaussian quadrature rules. Here $v-v_{h}, \phi-\phi_{h}$, and $f$ and $g$ are given directly, whereas the evaluation of $K f, V f^{\prime}$, and $V g$ again involves an integration over $\Gamma$ which is done approximately with a 32-point Gaussian quadrature rule. Finally, using $W=-\frac{\partial}{\partial s} V \frac{\partial}{\partial s}$ again [16], $W f$ and $W v_{h}$ are rewritten as derivatives of $-V f^{\prime}$ and $-V v_{h}^{\prime}$ computed by numerical differentiation with respect to the arc length.

The norms of the appearing residuals $\left\|R_{2}\right\|_{L^{2}\left(\Gamma_{j}\right)}$ and $\left\|R_{1}^{\prime}\right\|_{L^{2}\left(\Gamma_{j}\right)}$ are computed elementwise with a 10-point Gaussian quadrature rule, where the derivative in $\left\|R_{1}^{\prime}\right\|_{L^{2}\left(\Gamma_{j}\right)}$ is evaluated by numerical differentiation.

7.3. Numerical examples Let $\Omega_{1}$ be the L-shaped domain with vertices $(0,0)$, $(0,1),(-1,1),(-1,-1),(1,-1)$ and $(1,0)$. The data $f$ and $g$ in the transmission problem are given by (2) and

$$
u_{1}=r^{2 / 3} \cdot \sin \left(\frac{2}{3} \varphi\right), \quad u_{2}=\log \left|\left(x_{1}, x_{2}\right)+\frac{1}{2}(1,-1)\right|
$$

with polar coordinates $(r, \varphi)$ and Cartesian coordinates $\left(x_{1}, x_{2}\right)$.

We consider four adaptive algorithms as motivated in [4]. The first is considered for the present example in [3]. For a given "triangulation" of the boundary $\Gamma=\cup_{j=1}^{N} \Gamma_{j}$ we can compute an approximation of the contribution $a_{j}:=\left\|R_{1}^{\prime}\right\|_{L^{2}\left(\Gamma_{j}\right)}+\left\|R_{2}\right\|_{L^{2}\left(\Gamma_{j}\right)}$ of one element $\Gamma_{j}$ as explained in the previous subsections.

Then, ignoring the constant $c>0$, we have that the error in the energy norm is bounded by

$$
\left(\sum_{j=1}^{N} a_{j}^{2}\right)^{1 / 4} \cdot\left(\sum_{j=1}^{N} h_{j}^{2} \cdot a_{j}^{2}\right)^{1 / 4},
$$

where $h_{j}:=\left|\Gamma_{j}\right|$ is the length of the element $\Gamma_{j}$. Using this notation our numerical experiments are performed with the following algorithms (A1), (A2), (A3), and (A4) which depend on the parameters $\theta, 0 \leq \theta \leq 1$, and TOL $>0$.

Adaptive Algorithm ( $A k$ ).

Starting with a given quasi-uniform coarse initial mesh, take the actual partition $\Gamma_{1}, \ldots, \Gamma_{N}$, and perform (1)-(3) until termination.

(1) Solve the Galerkin equations with a trial space of piecewise polynomials with respect to the actual partition $\Gamma_{1}, \ldots, \Gamma_{N}$.

(2) Compute $a_{1}, \ldots, a_{N}, h_{1}, \ldots, h_{N}$ and the related quantities in (13). Decide: stop and terminate or refine and continue with (3).

(3) For $j=1, \ldots, N$ refine $\Gamma_{j}$ by the rule $(A k)$ and continue with the new mesh in (1) : 
(A1) Halve $\Gamma_{j}$ if and only if $a_{j} \cdot h_{j} \geq \theta \cdot \max _{k=1, \ldots, N} a_{k} \cdot h_{k}$.

(A2) Halve $\Gamma_{j}$ if and only if $a_{j} \cdot \sqrt{h_{j}} \geq \theta \cdot \max _{k=1, \ldots . N} a_{k} \cdot \sqrt{h_{k}}$.

(A3) Divide $\Gamma_{j}$ in $k_{j}$ pieces of the same length, where $k_{j}$ is the smallest integer $\geq 1$ with

$$
h_{j} / k_{j} \leq \frac{\mathrm{TOL}^{2}}{a_{j} \cdot \sqrt{N \cdot a}}, \quad a:=\sqrt{\sum_{j=1}^{N} a_{j}^{2}} .
$$

(A4) Divide $\Gamma_{j}$ in $k_{j}$ pieces of the same length, where $k_{j}$ is the smallest integer $\geq 1$ with

$$
h_{j} / k_{j} \leq\left(\mathrm{TOL} / N a_{j}\right)^{2} .
$$

REMARK 8. (i) Algorithms (A1) and (A2) are motivated by the goal to reach a uniform distribution of the element contributions $a_{j}$. Note that $\theta=0$ gives a uniform triangulation and with increasing $\theta$ the number of refined elements in the present step decreases. Algorithms (A3) and (A4) are motivated in the previous section.

(ii) Algorithms (A2) and (A4) can be justified connecting the results in [4] with Lemmas 3 and 5.

(iii) Although pseudodifferential operators are non-local we hope that a refinement of $\Gamma_{j}$ leads to improved bounds for the error.

The numerical results of the adaptive algorithms are shown in Table 1 for the uniform mesh and our four adaptive algorithms (A1), (A2), (A3), (A4) related to the paremeters $\theta=0.5, \theta=0.5$, TOL $=3.4$, and $\mathrm{TOL}=15.0$, respectively.

In Table 1, $N$ denotes the number of degrees of freedom (chosen by the algorithm; a new row corresponds to a new refinement step in the adaptive algorithm), $e_{N}$ denotes the corresponding relative error in the energy norm (see above) and $\alpha_{N}$ is the experimental convergence rate computed as

$$
\alpha_{N}=\log \left(e_{N^{\prime}} / e_{N}\right) / \log \left(N / N^{\prime}\right),
$$

where $N^{\prime}$ and $e_{N}^{\prime}$ are the corresponding values of the previous row. We define an experimental approximation for the constant $c>0$ in (10)

$$
\gamma_{N}:=\frac{\sqrt{\left\langle\phi-\phi_{h}, R_{1}\right\rangle+\left\langle v-v_{h}, R_{2}\right\rangle}}{\left(\sum_{j=1}^{N} a_{j}^{2}\right)^{1 / 4} \cdot\left(\sum_{j=1}^{N} h_{j}^{2} \cdot a_{j}^{2}\right)^{1 / 4}},
$$

where notation from Section 7.2 and Section 7.3 has been used. Values for $\gamma_{N}$ are given for the uniform meshes, (A1), and (A3) which are related to (10). The values $\gamma_{N}$ are bounded, as claimed in Theorem 5 .

From Table 1 we see also that a uniform mesh yields a convergence rate near the expected value $2 / 3$. The convergence achieved by the adaptive algorithm is improved: 
TABLE 1. Numerical results

\begin{tabular}{llll}
\hline \multicolumn{4}{l}{ Uniform mesh } \\
$N$ & $e_{N}$ & $\alpha_{N}$ & $\gamma_{N}$ \\
\hline 16 & 0.3802 & & .082 \\
32 & 0.1994 & 0.93 & .067 \\
64 & 0.1244 & 0.68 & .061 \\
128 & 0.0783 & 0.67 & .056 \\
256 & 0.0448 & 0.81 & .046 \\
\hline
\end{tabular}

\begin{tabular}{llll}
\hline \multicolumn{4}{c}{ (A1) for $\theta=0.5$} \\
$N$ & $e_{N}$ & $\alpha_{N}$ & $\gamma_{N}$ \\
\hline 16 & 0.3802 & & .082 \\
28 & 0.2122 & 1.04 & .063 \\
44 & 0.1322 & 1.05 & .054 \\
68 & 0.0806 & 1.14 & .043 \\
124 & 0.0458 & 0.94 & .034 \\
208 & 0.0232 & 1.31 & .022 \\
\hline
\end{tabular}

\begin{tabular}{lll}
\hline \multicolumn{3}{c}{ (A2) for $\theta=0.5$} \\
$N$ & $e_{N}$ & $\alpha_{N}$ \\
\hline 16 & 0.3802 & \\
28 & 0.2122 & 1.04 \\
44 & 0.1322 & 1.05 \\
64 & 0.0814 & 1.30 \\
104 & 0.0468 & 1.14 \\
184 & 0.0231 & 1.23 \\
\hline
\end{tabular}

\begin{tabular}{llll}
\hline \multicolumn{4}{c}{ (A3) for TOL $=3.4$} \\
$N$ & $e_{N}$ & $\alpha_{N}$ & $\gamma_{N}$ \\
\hline 16 & 0.3802 & & .082 \\
32 & 0.1678 & 1.18 & .053 \\
52 & 0.0820 & 1.48 & .036 \\
84 & 0.0462 & 1.19 & .025 \\
116 & 0.0184 & 2.85 & .012 \\
\hline
\end{tabular}

\begin{tabular}{lll}
\hline \multicolumn{3}{l}{ (A4) for TOL $=15.0$} \\
$N$ & $e_{N}$ & $\alpha_{N}$ \\
\hline 16 & 0.3802 & \\
20 & 0.2391 & 2.08 \\
28 & 0.1671 & 1.07 \\
32 & 0.1318 & 1.78 \\
52 & 0.0616 & 1.56 \\
84 & 0.0254 & 1.85 \\
\hline
\end{tabular}


for example, to obtain a relative error of 5/100 we need 256 degrees of freedom with a uniform mesh but only $124,108,84$ and 84 degrees of freedom using the adaptive algorithms with the mentioned parameters in 5, 5, 4 and 5 refinement steps. From this we conclude that the meshes obtained by our adaptive schemes are of high interest not only to improve asymptotic convergence rates but also to improve the error with only a few degrees of freedom. The most efficient algorithm in this example is (A3). The corresponding meshes for this situation are shown in Figure 1.

In order to compare the adaptive algorithms for various parameters the data is compressed using figures. Each of the four adaptive algorithms has a different figure, namely Figure 2 for (A1), Figure 3 for (A2), Figure 4 for (A3), and Figure 5 for (A4). In the figures an entry corresponds to a symbol (like $\Delta, \nabla, \diamond$ etc.) depending on the parameter. The entries belonging to the same parameter are connected by a straight line. The $x$-coordinate of a symbol is $\log (N)$, where $N$ is the number of degrees of freedom corresponding to a mesh, and the related Galerkin solution has a relative error $e_{N}$. Then, the $y$-coordinate of the symbol is $\log \left(e_{N}\right)$. However, the numbers shown on the axis are $e_{N}$ and $N$.

The values for TOL are chosen after some experiments. From Figures $2-5$ we see that the convergence rates are improved for all adaptive algorithms presented in this paper: the mean experimental convergence order for (A1) and (A2) is nearly 1.2 while the order for (A3) and (A4) approaches the optimal value 1.5.

7.4. Conclusion From the numerical experiments, we conclude that adaptive methods are important tools for an efficient numerical solution of transmission or interface problems. Compared with a coupling of finite elements and boundary elements, we claim from the experiments in [5] that the adaptive numerical treatment of the transmission problem via the boundary integral element method considered here is more efficient. The asymptotic convergence rates are improved as well as the quality of the Galerkin solutions corresponding to only a few degrees of freedom. This points to the efficiency of the adaptive algorithm and hence to the significance and sharpness of the a posteriori error estimate.

\section{Acknowledgement}

The authors thank S. Eicke for calculating the numerical examples and the DFG Forschergruppe at the University of Hannover for support. 

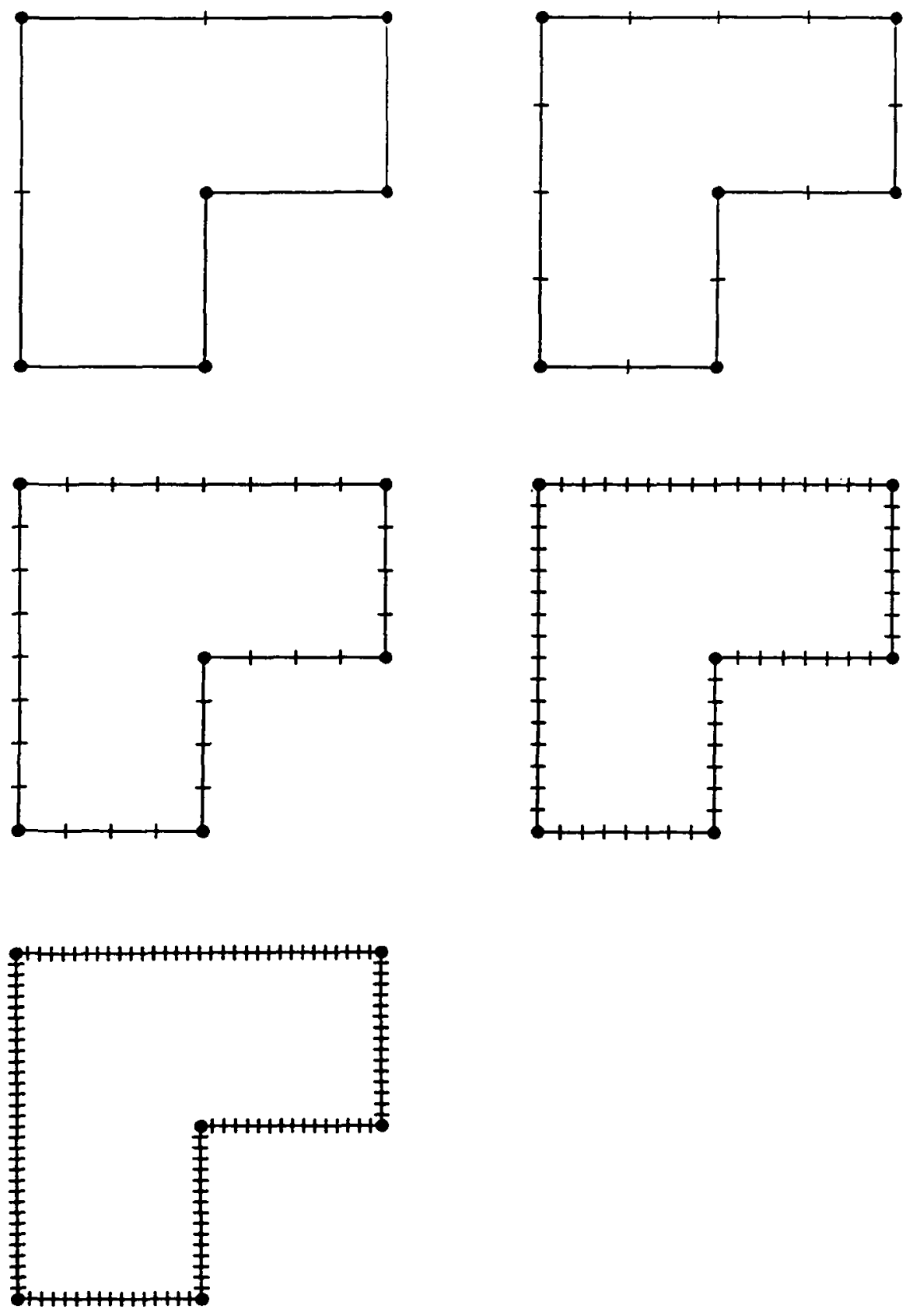

FIGURE 1A Uniform meshes 

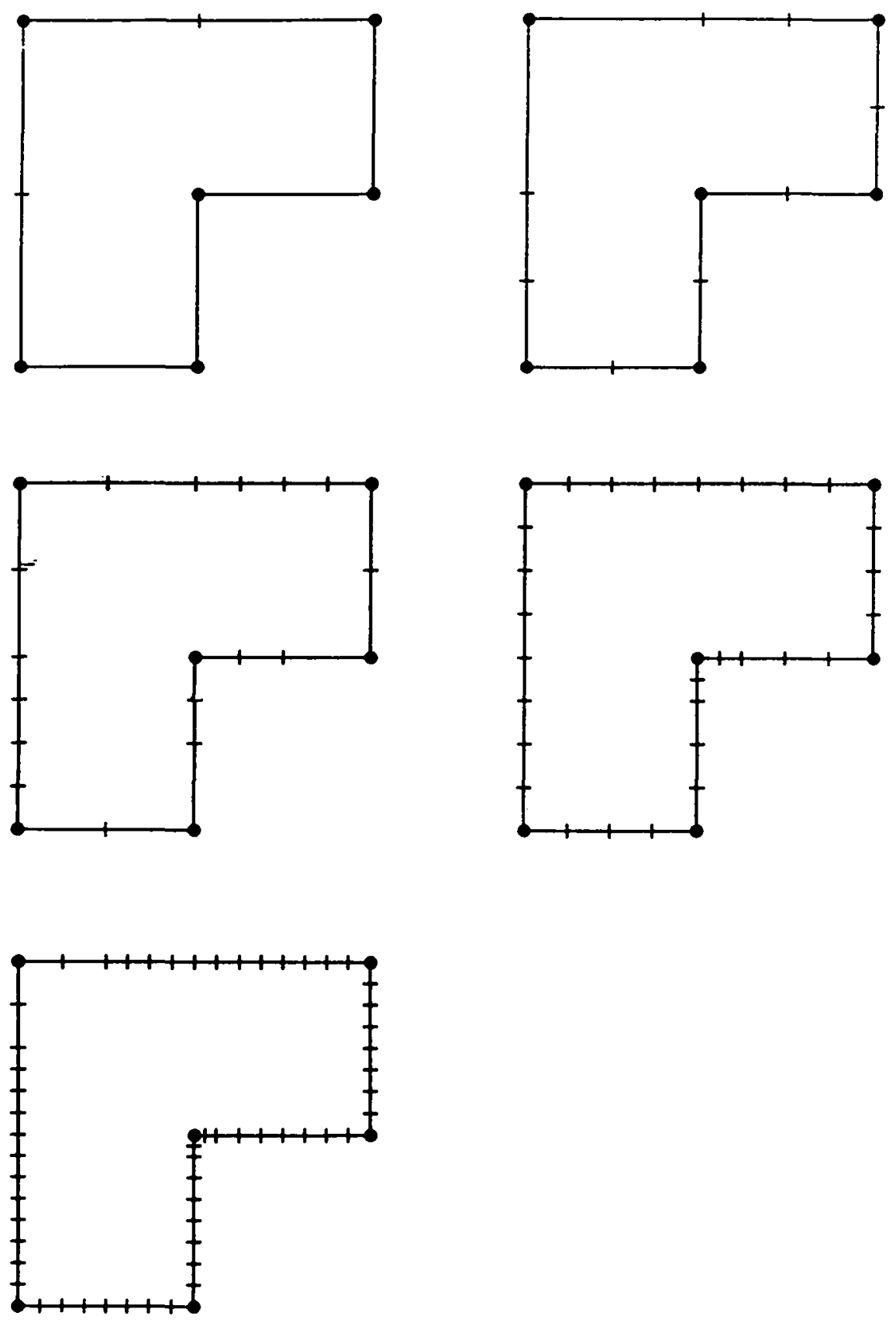

FIGURE 1B Meshes for A1, where $\theta=0.5$ 

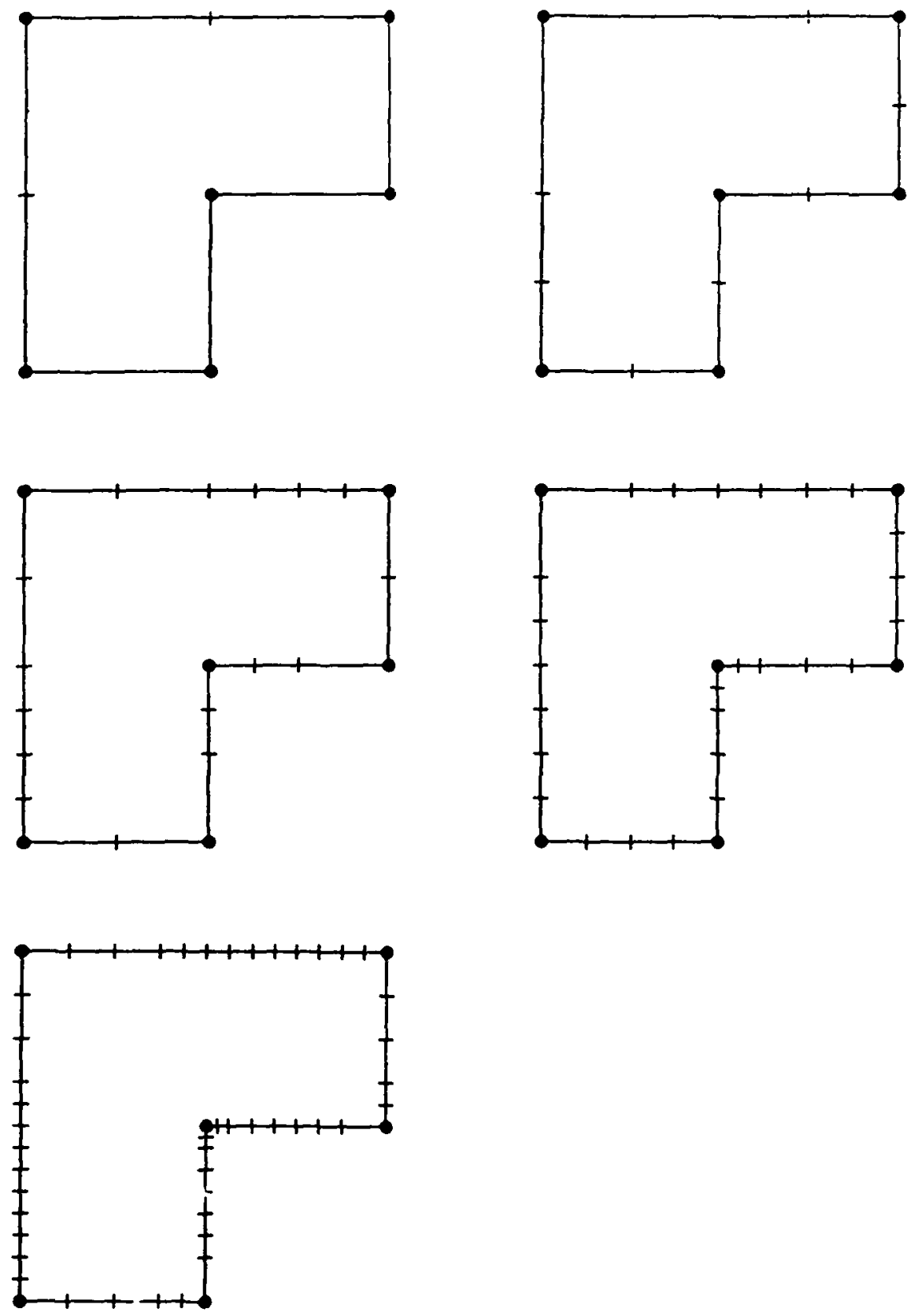

FIGURE 1C Meshes for A2, where $\theta=0.5$ 

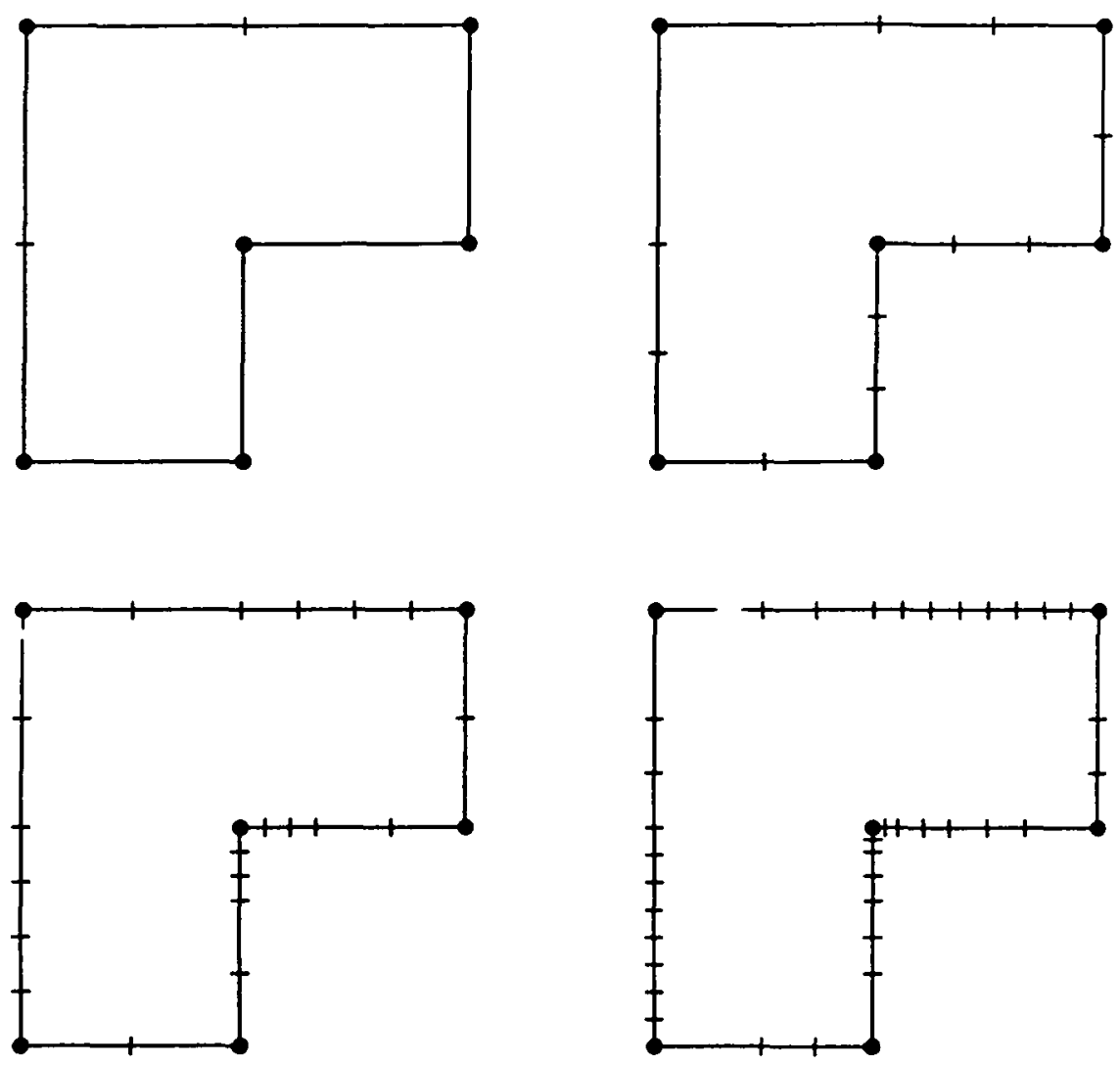

FIGURE 1D Meshes for A3, where TOL $=3.4$ 

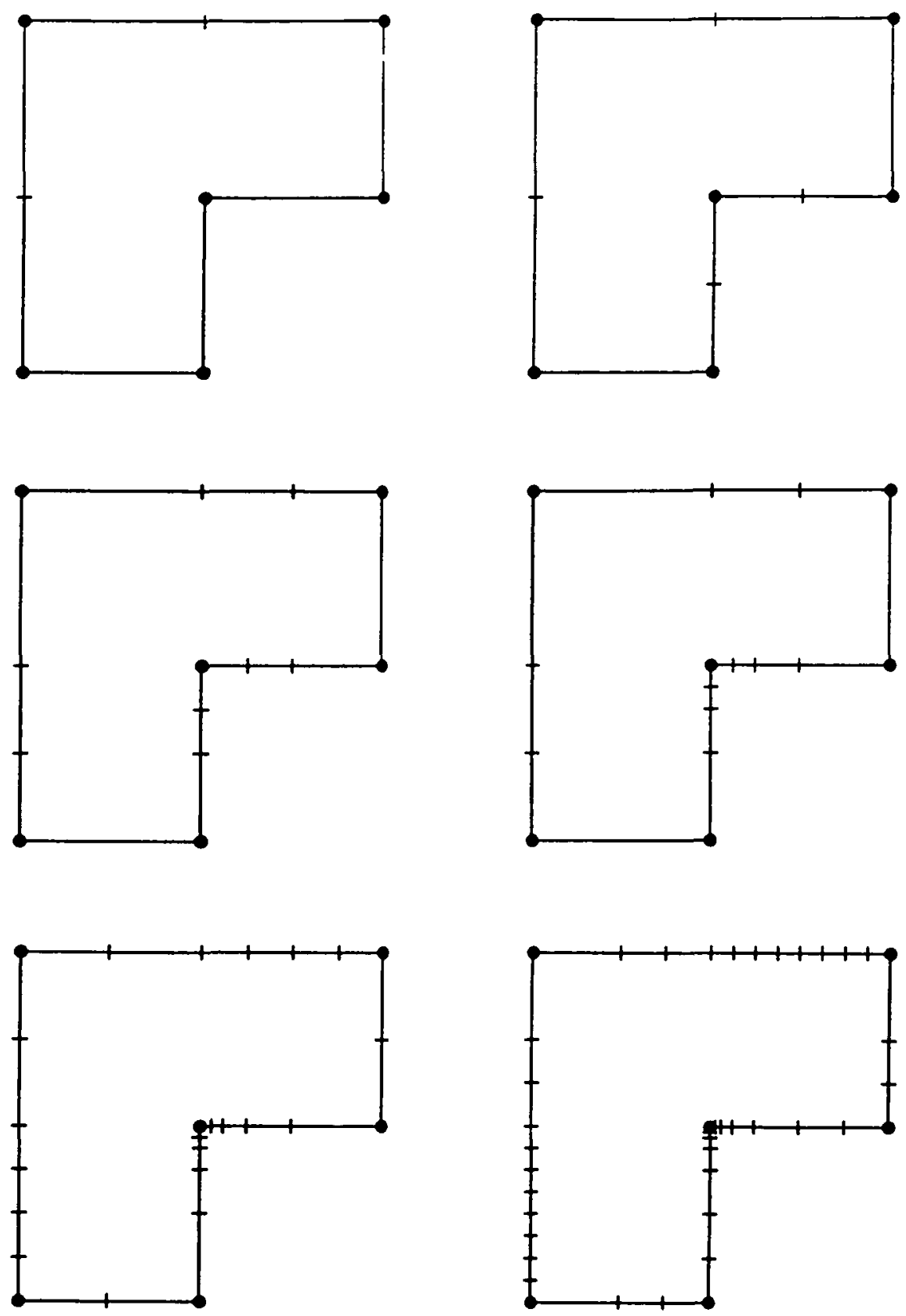

FIGURE lE Meshes for A4, where TOL $=3.0$ 


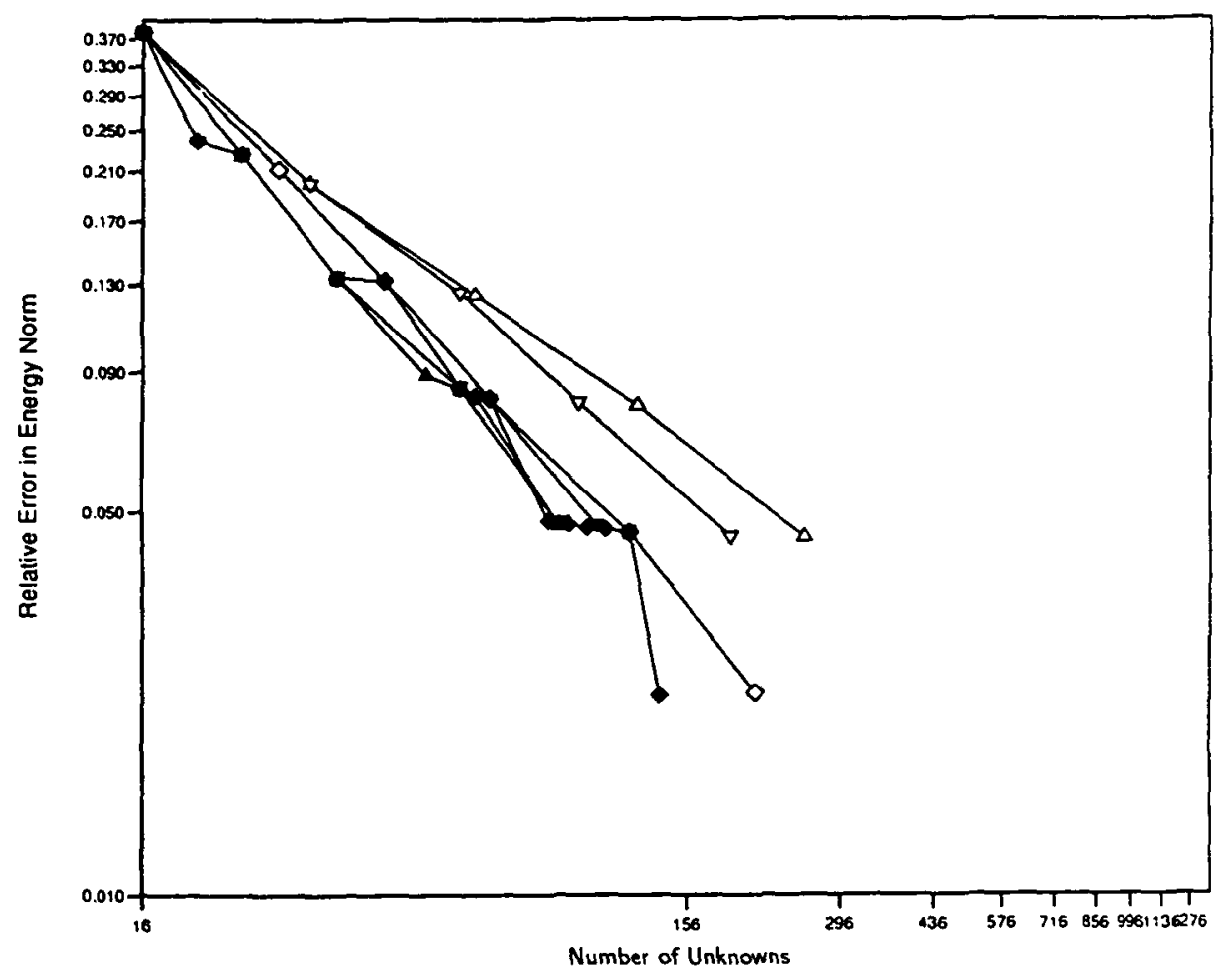

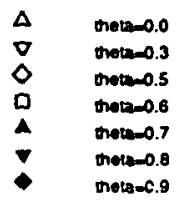

FIGURE 2. Error with Algorithm (Al) 


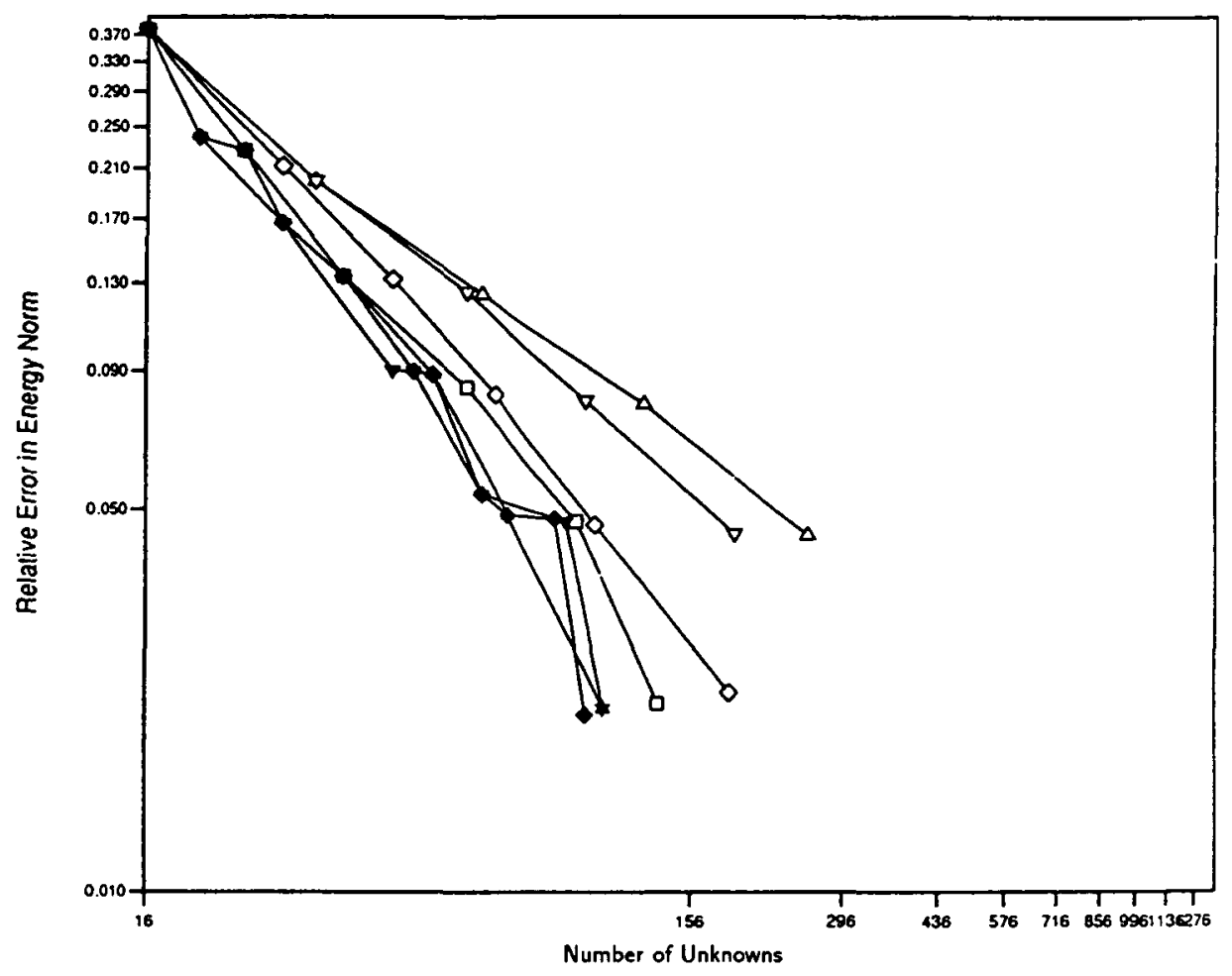

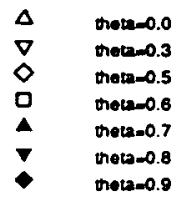

FIGURE 3. Error with Algorithm (A2) 


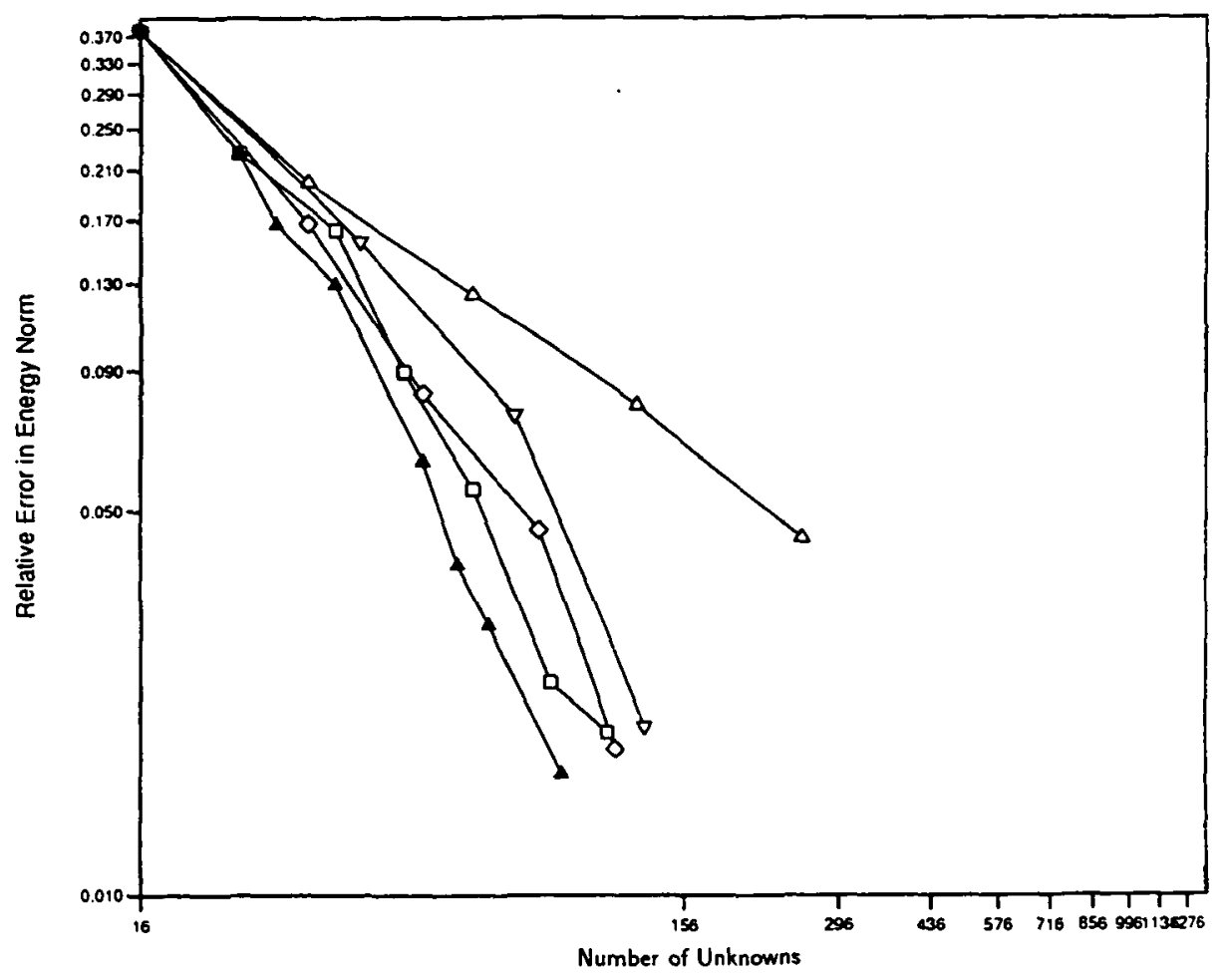

$\begin{array}{ll}\Delta & \text { Unitorm mesn } \\ \nabla & \text { TOL-3.0 } \\ 0 & \text { TOL }-3.4 \\ 0 & \text { TOL }-3.7 \\ \Delta & \text { TOL }-1.0\end{array}$

FIGURE 4. Error with Algorithm (A3) 


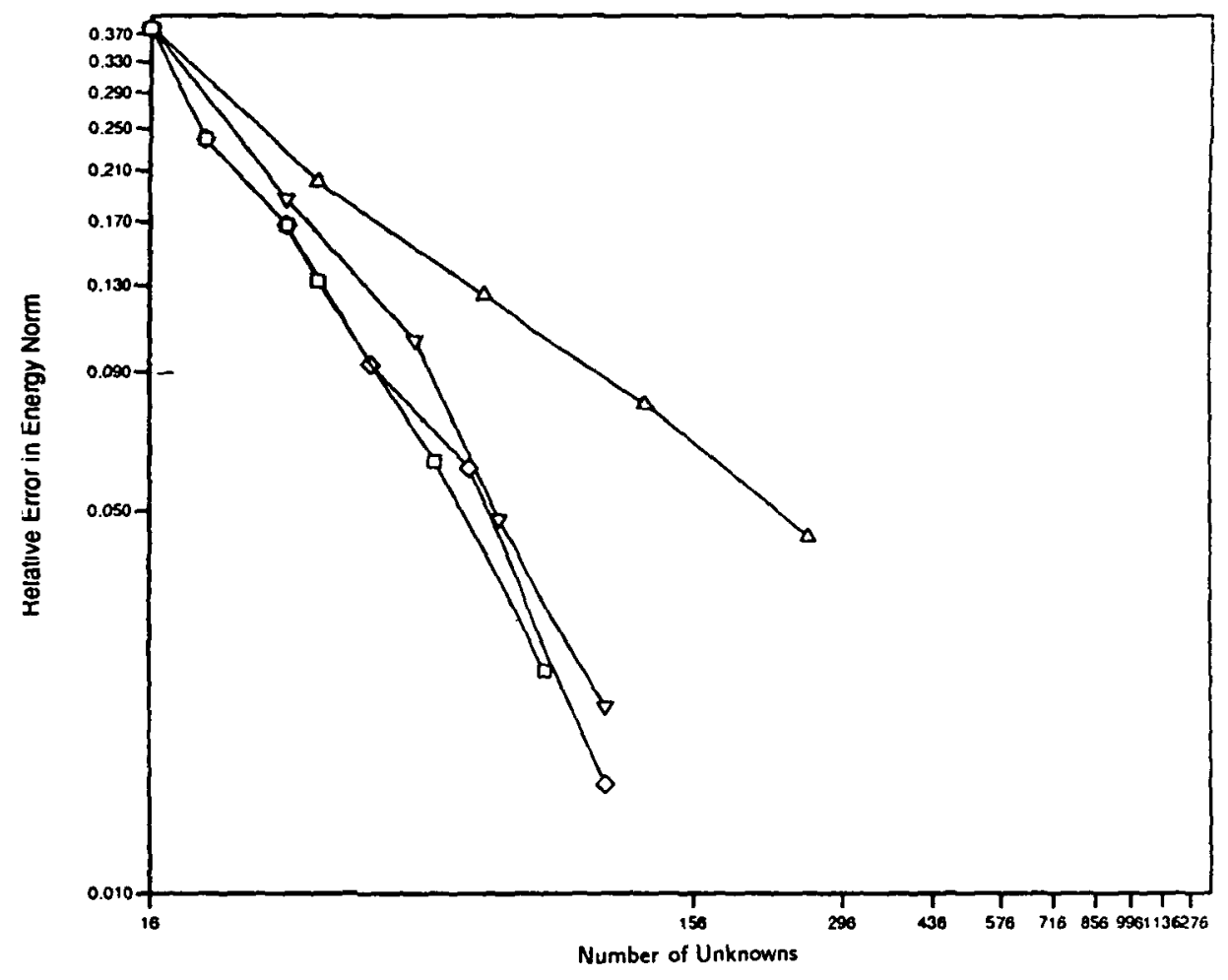

$\begin{array}{ll}\Delta & \text { Unitarm mosh } \\ \nabla & \text { TOL-10.0 } \\ \Delta & \text { roL-13.0 } \\ \square & \text { rol-15.0 }\end{array}$

FIGURE 5. Error with Algorithm (A4) 


\section{References}

[1] I. Babuska and A. Miller, "A posteriori error estimates and adaptive techniques for the finite element method", Tech. Note BN-968, (Univ. of Maryland, Institute for Physical Science and Technology, College Park, 1981.)

[2] J. Bergh and J. Löfström, Interpolation spaces (Springer, Berlin, 1976).

[3] C. Carstensen and E. P. Stephan, "Adaptive boundary element methods for some first kind integral equations", SIAM J. Numer. Anal. (1994), accepted for publication.

[4] C. Carstensen and E. P. Stephan, "A posteriori error estimates for boundary element methods", Math. Comp. 64 (1995) 483-500.

[5] C. Carstensen and E. P. Stephan, "Adaptive coupling of boundary elements and finite elements", Mathematical Modelling and Numerical Analysis 23 (1995) 779-817.

[6] M. Costabel, "Boundary integral operators on Lipschitz domains: Elementary results", SIAM J. Numer. Anal. 19 (1988) 613-626.

[7] M. Costabel and E. P. Stephan, "A direct boundary integral equation method for transmission problems", J. Math. Anal. Appl. 106 (1985) 367-413.

[8] M. Costabel and E. P. Stephan, "Integral equations for transmission problems in linear elasticity", J. Integr. Eq. Appl. 2 (1990) 211-223.

[9] M. Crouzeix and V. Thomee, "The stability in $L_{p}$ and $W_{p}^{1}$ of the $L_{2}$ - projection onto finite element function spaces", Math. Comp. 48 (1987) 521-532.

[10] K. Eriksson and C. Johnson, "An adaptive finite element method for linear elliptic problems", Math. Comp. 50 (1988) 361-383.

[11] K. Eriksson and C. Johnson, "Adaptive finite element methods for parabolic problems. I. A linear model problem", SIAM J. Numer. Anal. 28 (1991) 43-77.

[12] V. Ervin, N. Heuer and E. P. Stephan, "On the $h-p$ version of the boundary element method for Symm's integral equation on polygons", Comput. Meth. Appl. Mech. Engin. 110 (1993) 25-38.

[13] D. Gaier, "Integralgleichungen erster Art und konforme Abbildung", Math. Z. 147 (1976) 113-129.

[14] C. Johnson and P. Hansbo, "Adaptive finite element methods in computational mechanics", Comput. Meth. Appl. Mech. Engin. 101 (1992) 143-181.

[15] J. L. Lions and E. Magenes, Non-homogeneous boundary value problems and applications, Vol. I (Springer, Berlin, 1972).

[16] J. C. Nedelec, "La méthode des élements finis a apliquée aux equations intégrales de la physique", (First meeting AFCET-SMF on applied mathematics Palaiseau. Vol. 1, 181-190, 1978.).

[17] F. V. Postell and E. P. Stephan, "On the $h-, p-$ and $h-p$ versions of the boundary element method - numerical results", Comput. Meth. Appl. Mech. Engin. 83 (1990) 69-89.

[18] E. Rank, "Adaptive boundary element methods", in Boundary Elements 9, Vol.1 (eds. C. A. Brebbia, W. L. Wendland and G. Kunn), (Springer, Heidelberg, 1987) 259-273.

[19] 1. H. Sloan and A. Spence, "The Galerkin method for integral equations of the first kind with logarithmic kernel: Theory", IMA J. Numer. Anal. 8 (1988) 105-122. 